This is a self-archived version of an original article. This version may differ from the original in pagination and typographic details.

Author(s): Matculevich, Svetlana; Wolfmayr, Monika

Title: On the a posteriori error analysis for linear Fokker-Planck models in convectiondominated diffusion problems

Year: 2018

Version: Accepted version (Final draft)

Copyright: @ 2018 Elsevier Inc

Rights: In Copyright

Rights url: http://rightsstatements.org/page//nC/1.0/?language=en

Please cite the original version:

Matculevich, S., \& Wolfmayr, M. (2018). On the a posteriori error analysis for linear FokkerPlanck models in convection-dominated diffusion problems. Applied Mathematics and Computation, 339, 779-804. https://doi.org/10.1016/j.amc.2018.05.050 


\title{
On the a posteriori error analysis for linear Fokker-Planck models in convection-dominated diffusion problems
}

\author{
Svetlana Matculevich * $\quad$ Monika Wolfmayr ${ }^{\dagger}$
}

\begin{abstract}
This work is aimed at the derivation of reliable and efficient a posteriori error estimates for convectiondominated diffusion problems motivated by a linear Fokker-Planck problem appearing in computational neuroscience. We obtain computable error bounds of functional type for the static and time-dependent case and for different boundary conditions (mixed and pure Neumann boundary conditions). Finally, we present a set of various numerical examples including discussions on mesh adaptivity and space-time discretisation. The numerical results confirm the reliability and efficiency of the error estimates derived.
\end{abstract}

Keywords: a posteriori error estimation; convection-dominated diffusion problems; elliptic partial differential equations; parabolic partial differential equations; mesh-adaptivity.

\section{Introduction}

Convection-diffusion systems are widely used in natural sciences and financial mathematics for describing physical flows and stochastically changing systems, respectively. The Fokker-Planck (or Kolmogorov) equation is one of the examples of this class illustrating the velocity of a particle in the Brownian motion, along with the Black-Scholes equation governing the price evolution, and the Navier-Stokes equation that describes the motion of viscous fluid substances. There are many more problems appearing in physics or biology that are concerned with modelling of semiconductors or of decision-making processes in neuroscience that can be targeted by this type of systems. The considered equation is sometimes also called the drift-diffusion equation.

This paper derives and discusses a posteriori error estimates of the functional type for convection-diffusion problems, which are motivated by the decision-making Fokker-Planck model problem discussed by Carrillo et al. [15] appearing in computational neuroscience. The mathematical model discussed in [15] is associated with stochastic dynamical systems, modelling the evolution of the decision-making (average firing rates) of two interacting neurone populations, see also [13] and [17. In [15], the existence of a unique solution for stationary as well as evolutionary linear Fokker-Planck equations is discussed and proved under the assumption that the (regular enough) flux or drift is incoming in the bounded domain. The proofs are mainly based on the work by Wloka [56] as well as Evans [20]. In [56], a more general result is proved, which can be then applied in order to show existence and uniqueness in the time-dependent case too. Carrillo et al. [15] discusses this issue together with the large time behaviour of the problem and proves for that the convergence of the solution to the stationary state.

The main contribution of this work is the derivation of two-sided functional a posteriori error estimates (the so-called majorant and minorant) for stationary as well as time-dependent linear convection-diffusion problems. The ideas of functional type error bounds derivation were introduced by S. Repin in the 90's, see, e.g., 39, 38, 44, 40, 35. Naturally, there are alternative approaches in a posteriori error control such as the residual-based or the goal-oriented estimation techniques, see, e.g., the books [54, 3]. Moreover, we refer to the works [30, 55] dedicated to convectiondiffusion problems. Compared to other methods, the functional type a posteriori estimation techniques provide a general (only functional based) framework to derive independent and guaranteed upper bounds. The initial work addressing elliptic convection-diffusion problems was done in [41, where the convection-dominated aspect was considered in detail (see, e.g., 41, Section 4.3.]). The derivation of majorants for the time-dependent reactionconvection-diffusion problem has been discussed in [46]. However, the numerical aspects as well as properties of the majorant applied to this class of problems has not been studied so far. With the current work, we fill this gap and consider various aspects of two-sided error bounds in applications. In particular, we present a set of

*RICAM Linz, Johann Radon Institute, Linz, Austria

$\dagger$ Faculty of Information Technology, University of Jyväskylä, Jyväskylä, Finland 
numerical examples for the linear Fokker-Planck model problem for different parameters and boundary conditions. A specially interesting case is the convection-dominated (CD) setting of the problem, which may follow from various applications, such as the linearised Navier-Stokes equation with high Reynolds number or drift-diffusion equations of a semiconductor device modelling. Due to the small diffusion coefficient, the solution of the discussed problem has singularities in form of boundary or interior layers. The classical numerical approximations relying on equidistant meshes are not able to capture these layers unless the mesh contains an unacceptably large amount of nodes. If the mesh is not fine enough, the obtained approximations will include the non-physical oscillations polluting the data. The techniques developed to trigger the above described issue include the upwind scheme 22], streamline diffusion finite element method (SDFEM), also known as streamline-upwind/Petrov-Galerkin formulation (SUPG) [12, residual free bubble (RFB) functions [7, 8, 7, 9, 10, Galerkin Least Squares (GLS) 25, 2, 47, Continuous Interior Penalty (CIP) [19, Edge Stabilisation (ES) [14, exponential fitting 4, 11, 53, 57, discontinuous Galerkin methods [6, 24], and spurious oscillations at layers diminishing (SOLD) methods [27, 28. This list is by no means exhaustive, and there exist other techniques tackling this issue. In this work, we use the SUPG method introduced by Brooks and Hughes [12.

An alternative approach to handle the state Fokker-Planck boundary value problem, see later (12)- 13 , , relies on an adaptation (grading) of the underlying meshes in order to capture the boundary layers. The mesh adaptation for convection-dominated problems (CDPs) affects both the stability and the accuracy of the scheme. In practice, it was observed that once the accuracy is improved through the mesh adaption, the scheme gets stabilised as well (see, e.g., [5, 47, 58]). According to the numerical results presented in [31, 47, 58, and the theoretical justification for a model problem discussed in [47, 59, meshes can be adapted to boundary levels in such a way that even a standard finite element method can provide the optimal or quasi-optimal approximation property. However, the question of constructing such a grid, correctly adapted to the particular problem, still remains open. Moreover, in [51] the authors conclude that the stabilisation of the CDP scheme is necessary. Since a priori error analysis does not provide directions on mesh adaptation, an appropriate a posteriori error estimate, extracting corresponding information from the obtained numerical approximations, would be a crucial tool to construct desirable meshes. In this work's numerical examples, we perform an adaptive strategy based on a bulk marking criterion for selecting the elements which have to be refined, see 18 .

According to Sun et al. [51, most a posteriori estimators constructed for elliptic type equations do not work well for the convection-dominated problems. John [26] presents a thorough numerical comparison of some standard a posteriori error estimators (e.g., residual-based error estimator, Zienkiewicz-Zhu estimator and error estimator based on the solution of local Neumann problems) for CDPs. Moreover, it is shown that no error estimator performs equally good for all numerical examples due to the presence of the term $\frac{1}{\varepsilon}$ (see, e.g., Kang and Yu [29]). For $\varepsilon$ close to zero, the error indicator might indicate wrong regions for the refinement.

This work is structured as follows: In Section 2, the relevant function spaces as well as the stationary and time-dependent linear Fokker-Planck model problems are presented. The derivation of two-sided estimates for the stationary problems is discussed in Section 3 followed by the corresponding results for the time-dependent case in Section 4. Finally, first numerical results for various given data sets are presented and discussed in Section 5 .

\section{Function spaces and Fokker-Planck model problems}

This section presents a short summary of the functional spaces' definitions needed throughout the paper. We also state the model problem for the static and time-dependent cases, which will be addressed from the a posteriori error control point of view in the upcoming sections.

\subsection{Function Spaces}

Let $\Omega \subset \mathbb{R}^{d}, d=\{1,2,3\}$, be a bounded domain with Lipschitz boundary $\Gamma=\partial \Omega$, where $\bar{\Omega}$ is the closure of $\Omega$. We denote by $\Gamma_{N}$ and $\Gamma_{D}$ with $\Gamma=\Gamma_{N} \cup \Gamma_{D}$, meas ${ }_{d-1} \Gamma_{N}>0$ and meas ${ }_{d-1} \Gamma_{D}>0$, the parts of the boundary $\Gamma$, where Robin or Dirichlet boundary conditions (BCs) are imposed, respectively. In this work, we consider problems with mixed and Robin boundary conditions. We denote by $L_{2}(\Omega)$ the Banach space of all Lebesgue-measurable functions $v$ defined on $\Omega$ for which

$$
\|v\|_{\Omega}:=\left(\int_{\Omega}|v(x)|^{2} \mathrm{~d} x\right)^{1 / 2}<\infty .
$$


In addition, we define the following weighted $L_{2}$ norm:

$$
\|v\|_{\varepsilon, \Omega}:=\varepsilon\left(\int_{\Omega}|v(x)|^{2} \mathrm{~d} x\right)^{1 / 2} .
$$

We define the $H^{1}$ spaces

$$
V_{0}:=\left\{v \in H^{1}(\Omega) \mid v=0 \text { on } \Gamma_{D}\right\}
$$

and

$$
\tilde{V}:=\left\{v \in H^{1}(\Omega) \mid\{v\}_{\Omega}=0\right\}, \text { where }\{v\}_{\Omega}:=\frac{1}{|\Omega|} \int_{\Omega} v \mathrm{~d} x
$$

with underlying standard $L_{2}$ spaces. Here, the first space includes the homogeneous Dirichlet boundary conditions and the second one contains functions with zero mean in $\Omega$. Moreover, we introduce the space

$$
Y_{\mathrm{div}}\left(\Omega, \Gamma_{N}\right):=\left\{\boldsymbol{y} \in L_{2}\left(\Omega, \mathbb{R}^{d}\right) \mid \operatorname{div} \boldsymbol{y} \in L_{2}(\Omega), \boldsymbol{y} \cdot \mathbf{n} \in L_{2}\left(\Gamma_{N}\right)\right\}
$$

of vector-valued functions satisfying the identity

$$
\int_{\Omega} \operatorname{div}(\boldsymbol{y} w) \mathrm{d} x=\int_{\Omega}(\operatorname{div} \boldsymbol{y} w+\boldsymbol{y} \cdot \nabla w) \mathrm{d} x=\int_{\Gamma_{N}}(\boldsymbol{y} \cdot \mathbf{n}) w \mathrm{~d} s, \quad \forall w \in V_{0} .
$$

Let us also define the space

$$
L_{[0,1]}^{\infty}(\Omega):=\left\{\chi \in L^{\infty}(\Omega) \mid 0 \leq \chi \leq 1 \text { a.a. } \Omega\right\} .
$$

Let $(0, T), 0<T<+\infty$, be a given time interval. Then, we denote by $Q_{T}:=\Omega \times(0, T)$ and $S_{T}=\partial \Omega \times(0, T)$ the space-time cylinder and its lateral surface, respectively. We define the space

$$
H^{1,1}\left(Q_{T}\right)=\left\{u \in L^{2}\left(Q_{T}\right) \mid \nabla_{x} u \in\left[L^{2}\left(Q_{T}\right)\right]^{d}, \partial_{t} u \in L^{2}\left(Q_{T}\right)\right\}
$$

in space and time with zero mean in $\Omega$ for a.a $t \in(0, T)$ as follows

$$
\tilde{V}_{0}=\left\{v \in H^{1,1}\left(Q_{T}\right) \mid\{v\}_{\Omega}=0 \text { for a.a. } t \in(0, T)\right\} .
$$

Analogously to (4), we define the space

$$
\boldsymbol{y} \in Y_{\operatorname{div}}\left(Q_{T}\right):=\left\{\boldsymbol{y} \in L_{2}\left(0, T ; L_{2}\left(\Omega, \mathbb{R}^{d}\right)\right) \mid \operatorname{div} \boldsymbol{y} \in L_{2}\left(0, T ; L_{2}(\Omega)\right), \boldsymbol{y} \cdot \mathbf{n} \in L_{2}\left(0, T ; L_{2}(\Gamma)\right)\right\}
$$

by means of equation

$$
\int_{Q_{T}} \operatorname{div}(\boldsymbol{y} w) \mathrm{d} x \mathrm{~d} t=\int_{Q_{T}}(\operatorname{div} \boldsymbol{y} w+\boldsymbol{y} \cdot \nabla w) \mathrm{d} x \mathrm{~d} t=\int_{S_{T}} \boldsymbol{y} \cdot \mathbf{n} \mathrm{d} s \mathrm{~d} t, \quad \forall w \in \tilde{V}_{0} .
$$

Finally, we define several inequalities, and corresponding to them constants, that are used throughout the work. By $C_{\mathrm{F}}$ [21] we denote the constant in Friedrichs' inequality given as follows

$$
\|w\|_{Q_{T}} \leq C_{\mathrm{F}}\|\nabla w\|_{Q_{T}}, \quad \forall w \in H_{0}^{1,0}\left(Q_{T}\right):=\left\{w \in L_{2}\left(Q_{T}\right): \nabla_{x} w \in\left[L_{2}\left(Q_{T}\right)\right]^{d}, w=0 \text { on } \Sigma\right\} .
$$

The Poincaré inequality [36, 37] reads as

$$
\|w\|_{Q_{T}} \leq C_{\mathrm{P}}\|\nabla w\|_{Q_{T}}, \quad \forall w \in \tilde{H}_{0}^{1,0}\left(Q_{T}\right):=\left\{w \in H_{0}^{1,0}\left(Q_{T}\right):\{w\}_{\Omega}=0\right\} .
$$

Finally, the classic trace inequality is given by

$$
\|u\|_{L_{2}\left(S_{T}\right)} \leq C_{\mathrm{tr}}\|u\|_{H^{1}\left(Q_{T}\right)}, \quad \forall u \in C^{1}\left(\overline{Q_{T}}\right)
$$




\section{$2.2 \quad$ Fokker-Planck model problems}

The state Fokker-Planck boundary value problem (BVP) reads as follows

$$
\begin{aligned}
-\operatorname{div} \boldsymbol{p}+\operatorname{div}(\mathbf{F} u) & =f, & & x \in \Omega, \\
\boldsymbol{p} & =\varepsilon \nabla u, & & x \in \Omega,
\end{aligned}
$$

where $f \in L_{2}(\Omega)$. In the following, we consider the problem 12 - 13 for two cases, which differ with respect to (w.r.t.) the boundary conditions imposed on it. In the first problem, we impose mixed BCs of the form

$$
\begin{aligned}
(\mathbf{F} u-\boldsymbol{p}) \cdot \mathbf{n} & =0, & & x \in \Gamma_{N}, \\
u & =0, & & x \in \Gamma_{D},
\end{aligned}
$$

on $(12)-13$, and in the second one, Robin BCs as follows

$$
(\mathbf{F} u-\boldsymbol{p}) \cdot \mathbf{n}=0, \quad x \in \Gamma .
$$

Here, $\mathbf{n}$ denotes the vector of unit outward normal to $\partial \Omega$, and the function $\mathbf{F}$ satisfies the following regularity requirements

$$
\mathbf{F} \in L^{\infty}\left(\Omega, \mathbb{R}^{d}\right), \quad \operatorname{div} \mathbf{F} \in L^{\infty}(\Omega),
$$

and the incoming flux conditions

$$
\mathbf{F} \cdot \mathbf{n}<0 \quad \text { on } \quad \Gamma_{N}, \quad \text { and } \quad \mathbf{F} \cdot \mathbf{n}<0 \text { on } \Gamma,
$$

in the case of mixed and Robin BCs, respectively.

The time-dependent version of the problem $112-13$ with Robin boundary conditions is discussed in section 4 as well. It reads as follows: find $u \in H^{1,1}\left(Q_{T}\right)$ satisfying

$$
\begin{aligned}
u_{t}-\operatorname{div} \boldsymbol{p}+\operatorname{div}(\mathbf{F} u) & =f, & & (x, t) \in Q_{T}, \\
\boldsymbol{p} & =\varepsilon \nabla u, & & (x, t) \in Q_{T}, \\
u(x, 0) & =u_{0}, & & x \in \Omega, \\
(\mathbf{F} u-\boldsymbol{p}) \cdot \mathbf{n} & =0, & & (x, t) \in S_{T},
\end{aligned}
$$

where

$$
f \in L_{2}\left(Q_{T}\right), \quad \text { and } \quad u_{0} \in \tilde{V} .
$$

Function $\mathbf{F}$ satisfies 17 for a.a $t \in(0, T)$ and the incoming flux condition $\mathbf{F} \cdot \mathbf{n}<0$ on $S_{T}$.

\section{Two-sided estimates for the static case}

The current section is dedicated to the derivation of the majorant and minorant of the distance between any approximation function $v$ and the exact solution $u$ of static or time-dependent Fokker-Planck problem. Presented upper bounds can be viewed as a generalisation of the majorant studied in [41, Section 4.3.] and [46]. The derived lower bound of the error is a completely original result.

\subsection{Error majorant in case of mixed boundary conditions}

By testing $\sqrt{12}$ with a function $\eta \in V_{0}$, we arrive at the generalised formulation of (12) -13 with boundary conditions (14)- 15 : find $u \in V_{0}$ satisfying the integral identity

$$
a(u, \eta)=\langle f, \eta\rangle_{\Omega}, \quad \forall \eta \in V_{0},
$$

where the non-symmetric bilinear form $a(\cdot, \cdot)$ and the right-hand side (RHS) are given by

$$
a(u, \eta):=\varepsilon \int_{\Omega} \nabla u \cdot \nabla \eta \mathrm{d} x-\int_{\Omega}(\mathbf{F} u) \cdot \nabla \eta \mathrm{d} x \quad \text { and } \quad\langle f, \eta\rangle_{\Omega}:=\int_{\Omega} f \eta \mathrm{d} x,
$$


respectively. Assume that function $v \in V_{0}$ is the approximation of solution $u$ and the distance $e=u-v \in V_{0}$ between them is measured in terms of the norm

$$
\|e\|_{\mathrm{M}}^{2} \equiv\|e\|_{(\nu, \delta, \chi)}^{2}:=\nu\|\nabla e\|_{\Omega}^{2}+\|\delta e\|_{\Omega}^{2}+\|\chi e\|_{\Gamma}^{2},
$$

where $\nu:=\varepsilon$ is a positive weight parameter, and $\delta^{2}:=\frac{1}{2} \operatorname{div} \mathbf{F}$ and $\chi^{2}:=\left(-\frac{1}{2} \mathbf{F} \cdot \mathbf{n}\right)^{1 / 2}$ are positive weight functions. By transforming (24) and assuming $\eta=e$, we arrive at the following equality

$$
\varepsilon \int_{\Omega} \nabla e \cdot \nabla e \mathrm{~d} x-\int_{\Omega}(\mathbf{F} e) \cdot \nabla e \mathrm{~d} x=\int_{\Omega}(f e-\varepsilon \nabla v \cdot \nabla e+(\mathbf{F} v) \cdot \nabla e) \mathrm{d} x .
$$

Substituting the identity

$$
-\int_{\Omega}(\mathbf{F} e) \cdot \nabla e \mathrm{~d} x=\frac{1}{2}\left(\int_{\Omega} \operatorname{div} \mathbf{F} e^{2} \mathrm{~d} x-\int_{\Gamma_{N}}(\mathbf{F} \cdot \mathbf{n}) e^{2} \mathrm{~d} s\right)
$$

into (27), we obtain the relation known as 'error-identity' for $e \in V_{0}$

$$
\varepsilon \int_{\Omega}|\nabla e|^{2} \mathrm{~d} x+\frac{1}{2}\left(\int_{\Omega} \operatorname{div} \mathbf{F} e^{2} \mathrm{~d} x-\int_{\Gamma_{N}}(\mathbf{F} \cdot \mathbf{n}) e^{2} \mathrm{~d} s\right)=\int_{\Omega}(f e-\varepsilon \nabla v \cdot \nabla e+(\mathbf{F} v) \cdot \nabla e) \mathrm{d} x .
$$

Following the ideas exposed in monographs [35, 41, 33, we introduce an auxiliary vector-valued function $\boldsymbol{y} \in Y_{\text {div }}\left(\Omega, \Gamma_{N}\right)$ satisfying the identity (5). By means of substituting (5) into (29), we arrive at

$$
\begin{aligned}
\|e\|_{(\nu, \delta, \chi)}^{2}:=\|\nabla e\|_{\varepsilon, \Omega}^{2}+ & \|\delta e\|_{\Omega}^{2}+\|\chi e\|_{\Gamma_{N}}^{2} \\
& =\int_{\Omega}(f-\operatorname{div}(\mathbf{F} v)+\operatorname{div} \boldsymbol{y}) e \mathrm{~d} x+\int_{\Omega}(\boldsymbol{y}-\varepsilon \nabla v) \cdot \nabla e \mathrm{~d} x+\int_{\Gamma_{N}}((\mathbf{F} v-\boldsymbol{y}) \cdot \mathbf{n}) e \mathrm{~d} s .
\end{aligned}
$$

From now on, let $\mathbf{r}_{\mathrm{eq}}, \mathbf{r}_{\mathrm{d}}$, and $\mathbf{r}_{\Gamma_{\mathrm{N}}}$ denote the residuals in the RHS of 30

$$
\mathbf{r}_{\mathrm{eq}}:=f-\operatorname{div}(\mathbf{F} v)+\operatorname{div} \boldsymbol{y}, \quad \mathbf{r}_{\mathrm{d}}:=\boldsymbol{y}-\varepsilon \nabla v, \quad \text { and } \quad \mathbf{r}_{\Gamma_{\mathrm{N}}}:=(\mathbf{F} v-\boldsymbol{y}) \cdot \mathbf{n},
$$

which also denote the residuals of the equations $(12), \sqrt{13})$, and $(14)$, respectively. In order to estimate the RHS of (30), we apply the Hölder inequality, i.e.,

$$
\int_{\Omega} \mathbf{r}_{\mathrm{d}} \cdot \nabla e \mathrm{~d} x \leq\left\|\mathbf{r}_{\mathrm{d}}\right\|_{\varepsilon^{-1}, \Omega}\|\nabla e\|_{\varepsilon, \Omega} .
$$

For treatment of the terms $\mathbf{r}_{\text {eq }}$ and $\mathbf{r}_{\Gamma_{\mathrm{N}}}$, we introduce weight functions $\mu, \theta$ from the space $L_{[0,1]}^{\infty}(\Omega)$ in order to obtain robust majorants w.r.t. drastic changes in terms of $\operatorname{div} \mathbf{F}$ and $\mathbf{F} \cdot \mathbf{n}$. Then, the terms with residuals $\mathbf{r}_{\mathrm{eq}}$ and $\mathbf{r}_{\Gamma_{\mathrm{N}}}$ can be estimated as follows:

$$
\int_{\Omega} \mathbf{r}_{\text {eq }} e \mathrm{~d} x=\int_{\Omega} \mu \mathbf{r}_{\text {eq }} e \mathrm{~d} x+\int_{\Omega}(1-\mu) \mathbf{r}_{\text {eq }} e \mathrm{~d} x \leq\left\|\frac{\mu}{\delta} \mathbf{r}_{\text {eq }}\right\|_{\Omega}\|\delta e\|_{\Omega}+\frac{C_{\mathrm{F}}}{\sqrt{\varepsilon}}\left\|(1-\mu) \mathbf{r}_{\text {eq }}\right\|_{\Omega}\|\nabla e\|_{\varepsilon, \Omega}
$$

and

$$
\int_{\Omega} \mathbf{r}_{\Gamma_{\mathrm{N}}} e \mathrm{~d} x=\int_{\Omega} \theta \mathbf{r}_{\Gamma_{\mathrm{N}}} e \mathrm{~d} x+\int_{\Omega}(1-\theta) \mathbf{r}_{\Gamma_{\mathrm{N}}} e \mathrm{~d} x \leq\left\|\frac{\theta}{\chi} \mathbf{r}_{\Gamma_{\mathrm{N}}}\right\|_{\Omega}\|\chi e\|_{\Gamma_{\mathrm{N}}}+\frac{C_{\mathrm{tr}}}{\sqrt{\varepsilon}}\left\|(1-\theta) \mathbf{r}_{\Gamma_{\mathrm{N}}}\right\|_{\Gamma_{\mathrm{N}}}\|\nabla e\|_{\varepsilon, \Omega} .
$$

The resulting majorant follows from the collection of (32)-(34) obtained by means of Young's inequality:

$$
\begin{aligned}
\|e\|_{(\nu, \delta, \chi)}^{2} & \leq\left(\left\|\mathbf{r}_{\mathrm{d}}\right\|_{\varepsilon^{-1}, \Omega}+\frac{C_{\mathrm{F}}}{\sqrt{\varepsilon}}\left\|(1-\mu) \mathbf{r}_{\mathrm{eq}}\right\|_{\Omega}^{2}+\frac{C_{\mathrm{tr}}}{\sqrt{\varepsilon}}\left\|(1-\theta) \mathbf{r}_{\Gamma_{\mathrm{N}}}\right\|_{\Gamma_{N}}\right)^{2}+\left\|\frac{\mu}{\delta} \mathbf{r}_{\mathrm{eq}}\right\|_{\Omega}^{2}+\left\|\frac{\theta}{\chi} \mathbf{r}_{\Gamma_{\mathrm{N}}}\right\|_{\Omega}^{2} \\
& \leq\left(\frac{\beta}{2}\left\|\mathbf{r}_{\mathrm{d}}\right\|_{\varepsilon^{-1}, \Omega}^{2}+\frac{1}{2 \beta}\left(\frac{\zeta}{2} \frac{C_{\mathrm{F}}^{2}}{\varepsilon}\left\|(1-\mu) \mathbf{r}_{\mathrm{eq}}\right\|_{\Omega}^{2}+\frac{1}{2 \zeta} \frac{C_{\mathrm{tr}}^{2}}{\varepsilon}\left\|(1-\theta) \mathbf{r}_{\Gamma_{\mathrm{N}}}\right\|_{\Gamma_{N}}^{2}\right)\right)+\left\|\frac{\mu}{\delta} \mathbf{r}_{\mathrm{eq}}\right\|_{\Omega}^{2}+\left\|\frac{\theta}{\chi} \mathbf{r}_{\Gamma_{\mathrm{N}}}\right\|_{\Gamma_{N}}^{2},
\end{aligned}
$$

where $\beta$ and $\zeta$ are positive parameters. Therefore,

$$
\|e\|_{\mathrm{M}}^{2} \equiv\|e\|_{(\nu, \delta, \chi)}^{2} \leq \inf _{\boldsymbol{y} \in Y_{\text {div }}(\Omega)} \overline{\mathrm{M}}_{\Gamma_{N}, \Omega}^{2}(v, \boldsymbol{y} ; \beta, \zeta),
$$

where

$$
\overline{\mathrm{M}}_{\Gamma_{N}, \Omega}^{2}(v, \boldsymbol{y} ; \beta, \zeta):=\frac{1}{2}\left(\beta\left\|\mathbf{r}_{\mathrm{d}}\right\|_{\varepsilon^{-1}, \Omega}^{2}+\frac{1}{2 \beta}\left(\zeta \frac{C_{\mathrm{F}}^{2}}{\varepsilon}\left\|\mathbf{r}_{\mathrm{eq}}\right\|_{\Omega}^{2}+\frac{1}{\zeta} \frac{C_{\mathrm{tr}}^{2}}{\varepsilon}\left\|\mathbf{r}_{\Gamma_{\mathrm{N}}}\right\|_{\Gamma_{N}}^{2}\right)\right)+\left\|\frac{\mu}{\delta} \mathbf{r}_{\mathrm{eq}}\right\|_{\Omega}^{2}+\left\|\frac{\theta}{\chi} \mathbf{r}_{\Gamma_{\mathrm{N}}}\right\|_{\Gamma_{N}}^{2} .
$$




\subsection{Error majorant in case of Robin boundary conditions}

Next, we consider the state Fokker-Planck BVP (12)- 13 with Robin BCs 16 . By testing 12 with a function $\eta \in \tilde{V}$, we arrive at the generalized formulation of (12)-(13) with (16): find $u \in \tilde{V}$ satisfying the integral identity $a(u, \eta)=\langle f, \eta\rangle_{\Omega}$ for all $\eta \in \tilde{V}$, where $a(u, \eta)$ and $\langle f, \eta\rangle_{\Omega}$ are defined in (25). Assume that function $v \in \tilde{V}$ is the approximation of solution $u$ and the error $e=u-v \in \tilde{V}$ is measured in terms of the norm (26). By transforming $a(u, \eta)=\langle f, \eta\rangle_{\Omega}$ and assuming $\eta=e$, we arrive at the error-identity

$$
\varepsilon \int_{\Omega} \nabla e \cdot \nabla e \mathrm{~d} x+\frac{1}{2}\left(\int_{\Omega} \operatorname{div} \mathbf{F} e^{2} \mathrm{~d} x-\int_{\Gamma}(\mathbf{F} \cdot \mathbf{n}) e^{2} \mathrm{~d} s\right)=\int_{\Omega}(f e-\varepsilon \nabla v \cdot \nabla e+(\mathbf{F} v) \cdot \nabla e) \mathrm{d} x,
$$

which is similar to 29 . Analogously to the previous subsection, we introduce an auxiliary vector-valued function $\boldsymbol{y} \in Y_{\operatorname{div}}(\Omega, \Gamma)$, which yields the relation

$$
\|\nabla e\|_{\varepsilon, \Omega}^{2}+\frac{1}{2}\left\|(\operatorname{div} \mathbf{F})^{1 / 2} e\right\|_{\Omega}^{2}+\frac{1}{2}\left\|(-\mathbf{F} \cdot \mathbf{n})^{1 / 2} e\right\|_{\Gamma}^{2}=\int_{\Omega} \mathbf{r}_{\text {eq }} e \mathrm{~d} x+\int_{\Omega} \mathbf{r}_{\mathrm{d}} \cdot \nabla e \mathrm{~d} x+\int_{\Gamma}\left(\mathbf{r}_{\Gamma} \cdot \mathbf{n}\right) e \mathrm{~d} s .
$$

In order to estimate the RHS of (38), we apply the Hölder, Young, Poincaré, and trace inequalities leading to

$$
\|e\|_{\overline{\mathrm{M}}}^{2} \equiv\|e\|_{(\nu, \delta, \chi)}^{2} \leq \inf _{\boldsymbol{y} \in Y_{\mathrm{div}}(\Omega)} \overline{\mathrm{M}}_{\Gamma, \Omega}^{2}(v, \boldsymbol{y} ; \beta, \zeta),
$$

where

$$
\overline{\mathrm{M}}_{\Gamma, \Omega}^{2}(v, \boldsymbol{y}):=\frac{1}{2}\left(\beta\left\|\mathbf{r}_{\mathrm{d}}\right\|_{\varepsilon, \Omega}^{2}+\frac{1}{2 \beta}\left(\zeta \frac{C_{\mathrm{P}}^{2}}{\varepsilon}\left\|(1-\mu) \mathbf{r}_{\mathrm{eq}}\right\|_{\Omega}^{2}+\frac{1}{\zeta} \frac{C_{\mathrm{tr}}^{2}}{\varepsilon}\left\|(1-\chi) \mathbf{r}_{\Gamma_{\mathrm{N}}}\right\|_{\Gamma_{N}}^{2}\right)\right)+\left\|\frac{\mu}{\delta} \mathbf{r}_{\mathrm{eq}}\right\|_{\Omega}^{2}+\left\|\frac{\theta}{\chi} \mathbf{r}_{\Gamma_{\mathrm{N}}}\right\|_{\Gamma_{N}}^{2} .
$$

\subsection{Error minorant in case of mixed boundary conditions}

In the following, we derive a lower bound of the approximation error in terms of a different quantity.

Theorem 1 For $v \in V_{0}$, the following inequality holds:

$$
\sup _{w \in V_{0}} \underline{\mathrm{M}}_{\Omega}^{2}(v, w):=\|e\|_{\underline{\underline{\mathrm{M}}}}^{2} \equiv\|e\|_{(\tilde{\nu}, \tilde{\delta}, \chi)}^{2},
$$

where $\tilde{\nu}=\frac{\varepsilon}{2}, \tilde{\delta}^{2}=\frac{1}{2}\left(\frac{|\mathbf{F}|^{2}}{\varepsilon}+\operatorname{div} \mathbf{F}\right), \chi^{2}=-\frac{1}{2} \mathbf{F} \cdot \mathbf{n}$, and

$$
\underline{\mathrm{M}}_{\Omega}^{2}(v, w):=\int_{\Omega}(f w-\varepsilon \nabla v \cdot \nabla w+\mathbf{F} v \cdot \nabla w) \mathrm{d} x-\frac{1}{2}\|\nabla w\|_{\varepsilon, \Omega}^{2} .
$$

Proof: The supremum can be estimated as follows

$$
\begin{aligned}
\sup _{w \in V_{0}}\left\{\int_{\Omega}(f w\right. & \left.-\varepsilon \nabla v \cdot \nabla w+\mathbf{F} v \cdot \nabla w) \mathrm{~d} x-\frac{\varepsilon}{2}\|\nabla w\|_{\Omega}^{2}\right\} \\
& =\sup _{w \in V_{0}}\left\{\int_{\Omega}(\varepsilon \nabla(u-v)-\mathbf{F}(u-v)) \cdot \nabla w \mathrm{~d} x-\frac{\varepsilon}{2}\|\nabla w\|_{\Omega}^{2}\right\} \\
& \leq \sup _{\tau \in L^{2}(\Omega)}\left\{\int_{\Omega}(\varepsilon \nabla e-\mathbf{F} e) \cdot \tau \mathrm{d} x-\frac{\varepsilon}{2}\|\tau\|_{\Omega}^{2}\right\} \\
& \leq \frac{1}{2 \varepsilon}\|\varepsilon \nabla e-\mathbf{F} e\|_{\Omega}^{2}=\frac{\varepsilon}{2}\|\nabla e\|_{\Omega}^{2}+\frac{1}{2 \varepsilon}\|\mathbf{F} e\|_{\Omega}^{2}-\int_{\Omega} \nabla e \cdot \mathbf{F} e \mathrm{~d} x .
\end{aligned}
$$

Substituting identity $(28)$ then yields the following estimate:

$$
\begin{aligned}
\sup _{w \in V_{0}}\left\{\int_{\Omega}(f w\right. & \left.-\varepsilon \nabla v \cdot \nabla w+\mathbf{F} v \cdot \nabla w) \mathrm{~d} x-\frac{1}{2}\|\nabla w\|_{\varepsilon, \Omega}^{2}\right\} \\
& \leq \frac{1}{2}\|\nabla e\|_{\varepsilon, \Omega}^{2}+\frac{1}{2 \varepsilon}\|\mathbf{F} e\|_{\Omega}^{2}+\frac{1}{2}\left\|(\operatorname{div} \mathbf{F})^{1 / 2} e\right\|_{\Omega}^{2}+\frac{1}{2}\left\|(-\mathbf{F} \cdot \mathbf{n})^{1 / 2} e\right\|_{\Gamma}^{2} \\
& =\frac{1}{2}\|\nabla e\|_{\varepsilon, \Omega}^{2}+\frac{1}{2}\left\|\left(\frac{|\mathbf{F}|^{2}}{\varepsilon}+\operatorname{div} \mathbf{F}\right)^{1 / 2} e\right\|_{\Omega}^{2}+\frac{1}{2}\left\|(-\mathbf{F} \cdot \mathbf{n})^{1 / 2} e\right\|_{\Gamma}^{2},
\end{aligned}
$$

which finally leads to equation (41). 
Remark 1 Note that the new norm differs from the norm $\|e\|_{(\nu, \delta, \chi)}^{2}$ only by the weighting parameters that are given by $\tilde{\nu}=\frac{\nu}{2}$ and $\tilde{\delta}^{2}=\delta^{2}+\frac{|\mathbf{F}|^{2}}{2 \varepsilon}$. The relation can also be written as follows

$$
\|e\|_{(\tilde{\nu}, \tilde{\delta}, \chi)}^{2}=\|e\|_{(\nu, \delta, \chi)}^{2}+\frac{1}{2 \varepsilon}\|\mathbf{F} e\|_{\Omega}^{2}-\frac{\varepsilon}{2}\|\nabla e\|_{\Omega}^{2} .
$$

When maximising the minorant functional, the auxiliary function $w \in V_{0}$ must be chosen from the richer approximation space in comparison to the approximation $v \in V_{0}$. Otherwise, the minorant vanishes. Analogously to the majorant functional, the minorant includes only known data and, therefore, is fully computable. By an appropriate selection of $w$, we can find a lower bound arbitrarily close to the exact error. In particular, if $w=u-v$, the minorant coincides with the error norm.

\section{Two-sided estimates for the time-dependent case}

\subsection{Upper estimates for the time-dependent case}

We consider now the parabolic initial-boundary value problem. After multiplying $(19)$ by a test function $\eta \in \tilde{V}_{0}$, we arrive at the generalized formulation of $19-22$ : find $u \in \tilde{V}_{0}$ satisfying the integral identity

$$
\begin{aligned}
\int_{\Omega}((u \eta)(x, T)-(u \eta)(x, 0)) \mathrm{d} x+\int_{Q_{T}} u \eta_{t} \mathrm{~d} x \mathrm{~d} t+\int_{Q_{T}} \varepsilon \nabla u \cdot \nabla \eta \mathrm{d} x \mathrm{~d} t-\int_{Q_{T}}(\mathbf{F} u) \cdot \nabla \eta \mathrm{d} x \mathrm{~d} t & \\
& =\int_{Q_{T}} f \eta \mathrm{d} x \mathrm{~d} t, \quad \forall \eta \in \tilde{V}_{0} .
\end{aligned}
$$

We present the functional error estimate, which provides a guaranteed upper bound of the deviation $e=u-v$ between the generalised solution of 46 and function $v \in \tilde{V}_{0}$ (generated by any numerical method) measured in terms of the norm

$$
[e]_{(\nu, \theta, \chi, \zeta)}^{2} \equiv[e] \frac{2}{\mathrm{M}, Q_{T}}:=\nu\|\nabla e\|_{Q_{T}}^{2}+\|\theta e\|_{Q_{T}}^{2}+\|\chi e\|_{S_{T}}^{2}+\zeta\|e(\cdot, T)\|_{\Omega}^{2},
$$

where $\nu$ and $\zeta$ are positive weight parameters, and $\theta, \chi$ are weight functions.

The initial step in the derivation of both upper estimates is the transformation of $(46)$ into the integral identity

$$
\begin{aligned}
\int_{\Omega}((e \eta)(x, T)-(e \eta)(x, 0)) \mathrm{d} x+\int_{Q_{T}} e \eta_{t} \mathrm{~d} x \mathrm{~d} t+\int_{Q_{T}} \varepsilon \nabla e \cdot \nabla \eta \mathrm{d} x \mathrm{~d} t-\int_{Q_{T}}(\mathbf{F} e) \cdot \nabla \eta \mathrm{d} x \mathrm{~d} t \\
\quad=\int_{Q_{T}}\left(f \eta+v_{t} \eta+(\mathbf{F} v) \cdot \nabla \eta-\varepsilon \nabla v \cdot \nabla \eta\right) \mathrm{d} x \mathrm{~d} t .
\end{aligned}
$$

By substituting $\eta=e$ and using the relation

$$
\int_{\Omega}((e \eta)(x, T)-(e \eta)(x, 0)) \mathrm{d} x+\int_{Q_{T}} e \eta_{t} \mathrm{~d} x \mathrm{~d} t=\frac{1}{2}\left(\|e(\cdot, T)\|_{\Omega}^{2}-\|e(\cdot, 0)\|_{\Omega}^{2}\right)
$$

we obtain

$$
\begin{aligned}
\frac{1}{2}\left(\|e(\cdot, T)\|_{\Omega}^{2}-\|e(\cdot, 0)\|_{\Omega}^{2}\right)+\varepsilon \int_{Q_{T}}|\nabla e|^{2} \mathrm{~d} x \mathrm{~d} t-\int_{Q_{T}}(\mathbf{F} e) \cdot \nabla e \mathrm{~d} x \mathrm{~d} t \\
\quad=\int_{Q_{T}}\left(f e+v_{t} e-\operatorname{div}(\mathbf{F} v) e-\varepsilon \nabla v \cdot \nabla e\right) \mathrm{d} x \mathrm{~d} t+\int_{S_{T}}(\mathbf{F} v) \cdot \mathbf{n} e \mathrm{~d} s \mathrm{~d} t
\end{aligned}
$$

Taking into account the equality

$$
-\int_{Q_{T}}(\mathbf{F} e) \cdot \nabla e \mathrm{~d} x \mathrm{~d} t=\frac{1}{2}\left(\int_{Q_{T}} \operatorname{div} \mathbf{F} e^{2} \mathrm{~d} x \mathrm{~d} t-\int_{S_{T}}(\mathbf{F} \cdot \mathbf{n}) e^{2} \mathrm{~d} s \mathrm{~d} t\right),
$$


we rewrite the LHS of 50 as follows

$$
\begin{aligned}
\frac{1}{2}\left(\|e(\cdot, T)\|_{\Omega}^{2}-\|e(\cdot, 0)\|_{\Omega}^{2}\right)+ & \varepsilon \int_{Q_{T}}|\nabla e|^{2} \mathrm{~d} x \mathrm{~d} t+\frac{1}{2}\left(\int_{Q_{T}} \operatorname{div} \mathbf{F} e^{2} \mathrm{~d} x \mathrm{~d} t+\int_{S_{T}}(-\mathbf{F} \cdot \mathbf{n}) e^{2} \mathrm{~d} s \mathrm{~d} t\right) \\
& =\int_{Q_{T}}\left(\left(f e-v_{t} e-\nabla(\mathbf{F} v) e\right) \mathrm{d} x \mathrm{~d} t-\varepsilon \nabla v \cdot \nabla e\right) \mathrm{d} x \mathrm{~d} t+\int_{S_{T}}(\mathbf{F} v) \cdot \mathbf{n} e \mathrm{~d} s \mathrm{~d} t .
\end{aligned}
$$

We follow the ideas from [45] and introduce an arbitrary vector-valued function $\boldsymbol{y} \in Y_{\text {div }}\left(Q_{T}\right)$ leading to the equation

$$
\begin{aligned}
\frac{1}{2}\left(\|e(\cdot, T)\|_{\Omega}^{2}\right. & \left.-\|e(\cdot, 0)\|_{\Omega}^{2}\right)+\varepsilon \int_{Q_{T}}|\nabla e|^{2} \mathrm{~d} x \mathrm{~d} t+\frac{1}{2}\left(\int_{Q_{T}} \operatorname{div} \mathbf{F} e^{2} \mathrm{~d} x \mathrm{~d} t+\int_{S_{T}}(-\mathbf{F} \cdot \mathbf{n}) e^{2} \mathrm{~d} s \mathrm{~d} t\right) \\
& =\int_{Q_{T}}\left(f-v_{t}-\nabla(\mathbf{F} v)+\operatorname{div} \boldsymbol{y}\right) e \mathrm{~d} x \mathrm{~d} t+\int_{Q_{T}}(\boldsymbol{y}-\varepsilon \nabla v) \cdot \nabla e \mathrm{~d} x \mathrm{~d} t+\int_{S_{T}}(\mathbf{F} v-\boldsymbol{y}) \cdot \mathbf{n} e \mathrm{~d} s \mathrm{~d} t .
\end{aligned}
$$

The residuals

$$
\mathbf{r}_{\mathrm{eq}}=f-v_{t}-\nabla(\mathbf{F} v)+\operatorname{div} \boldsymbol{y}, \quad \mathbf{r}_{\mathrm{d}}=\boldsymbol{y}-\varepsilon \nabla v, \quad \mathbf{r}_{\mathrm{ST}}=(\mathbf{F} v-\boldsymbol{y}) \cdot \mathbf{n}
$$

in the RHS of (53) correspond in a natural manner to the equations $\sqrt{19}),(20)$, and $(22)$, respectively. We proceed by estimating the terms in 53 using the Hölder, Young, Friedrichs as well as trace inequality, i.e.,

$$
\begin{gathered}
\int_{Q_{T}} \mathbf{r}_{\mathrm{eq}} e \mathrm{~d} x \mathrm{~d} t \leq\left\|\mathbf{r}_{\mathrm{eq}}\right\|_{Q_{T}}\|e\|_{\varepsilon, Q_{T}} \leq \frac{\alpha_{1}}{2} \frac{C_{\mathrm{F} \Omega}^{2}}{\varepsilon}\left\|\mathbf{r}_{\mathrm{eq}}\right\|_{Q_{T}}^{2}+\frac{1}{2 \alpha_{1}}\|\nabla e\|_{\varepsilon, Q_{T}}^{2}, \\
\int_{Q_{T}} \mathbf{r}_{\mathrm{d}} \cdot \nabla e \mathrm{~d} x \mathrm{~d} t \leq\left\|\mathbf{r}_{\mathrm{d}}\right\|_{Q_{T}}\|\nabla e\|_{\varepsilon, Q_{T}} \leq \frac{\alpha_{2}}{2}\left\|\mathbf{r}_{\mathrm{d}}\right\|_{\varepsilon^{-1}, Q_{T}}^{2}+\frac{1}{2 \alpha_{2}}\|\nabla e\|_{\varepsilon, Q_{T}}^{2}, \\
\int_{Q_{T}} \mathbf{r}_{\mathrm{S}_{\mathrm{T}}} e \mathrm{~d} s \mathrm{~d} t \leq\left\|\mathbf{r}_{\mathrm{S}_{\mathrm{T}}}\right\|_{\mathrm{S}_{\mathrm{T}}}\|e\|_{S_{T}} \leq \frac{\alpha_{3}}{2} \frac{C_{\mathrm{tr}}^{2}}{\varepsilon}\left\|\mathbf{r}_{\mathrm{S}_{\mathrm{T}}}\right\|_{S_{T}}^{2}+\frac{1}{2 \alpha_{3}}\|\nabla e\|_{\varepsilon, Q_{T}}^{2},
\end{gathered}
$$

where functions $\alpha_{i}>0, i=1,2,3$. Finally, we arrive at the estimate

$$
\begin{aligned}
\|e(\cdot, T)\|_{\Omega}^{2}+\left(2-\sum_{i=1}^{3} \frac{1}{\alpha_{i}}\right)\|\nabla e\|_{\varepsilon, Q_{T}}^{2}+\left\|(\operatorname{div} \mathbf{F})^{1 / 2} e\right\|_{Q_{T}}^{2}+\left\|(-\mathbf{F} \cdot \mathbf{n})^{1 / 2} e\right\|_{S_{T}}^{2}=:[e] \frac{\mathrm{M}, Q_{T}}{2} \\
\leq \overline{\mathrm{M}}_{Q_{T}}^{2}:=\|e(\cdot, 0)\|_{\Omega}^{2}+\left(\alpha_{1} \frac{C_{\mathrm{F} \Omega}^{2}}{\varepsilon}\left\|\mathbf{r}_{\mathrm{eq}}\right\|_{Q_{T}}^{2}+\alpha_{2}\left\|\mathbf{r}_{\mathrm{d}}\right\|_{\varepsilon^{-1}, Q_{T}}^{2}+\alpha_{3} \frac{C_{\mathrm{tr}}^{2}}{\varepsilon}\left\|\mathbf{r}_{\mathrm{S}_{\mathrm{T}}}\right\|_{S_{T}}^{2}\right),
\end{aligned}
$$

where parameter $\delta \in(0,2]$.

\subsection{Lower estimates for the time-dependent case}

Minorants of the error between the approximate and exact solutions for the evolutionary reaction-convectiondiffusion problem provide useful information while testing upper error-bounds (when $u$ is not available). The quality of the majorant is evaluated by considering its ratio w.r.t. the minorant. The first results related to lower error bounds for evolutionary problems were derived and numerically tested in [34. The result presented in the current section can be viewed as generalisation of Section 3 in [34.

Theorem 2 For any $v, \eta \in \tilde{V}_{0}$ the following estimate holds:

$$
\begin{aligned}
\sup _{\eta \in \tilde{V}_{0}} \underline{\mathrm{M}}^{2}(v, \eta) & :=\sup _{\eta \in \tilde{V}_{0}}\left\{\sum_{i=1}^{3} G_{i}(v, \eta)+F_{f u_{0}}(\eta)\right\} \\
& \leq[e]_{\underline{\mathrm{M}, Q_{T}}}^{2}:=\frac{1}{2}\|\nabla e\|_{\varepsilon, Q_{T}}^{2}+\frac{1}{2}\left\|\left(\frac{|\mathbf{F}|}{\varepsilon}+\operatorname{div} \mathbf{F}+1\right)^{1 / 2} e\right\|_{Q_{T}}^{2}+\frac{1}{2}\left\|(-\mathbf{F} \cdot \mathbf{n})^{1 / 2} e\right\|_{S_{T}}^{2}+\frac{1}{2}\|e(x, T)\|_{\Omega}^{2},
\end{aligned}
$$


where

$$
\begin{aligned}
G_{1}(v, \eta) & :=\int_{Q_{T}}\left(-\nabla \eta \cdot(\varepsilon \nabla v+\mathbf{F} v)-\frac{\varepsilon}{2}|\nabla \eta|^{2}\right) \mathrm{d} x \mathrm{~d} t, \\
G_{2}(v, \eta) & :=\int_{Q_{T}}\left(\eta_{t} v-\frac{1}{2}\left|\eta_{t}\right|^{2}\right) \mathrm{d} x \mathrm{~d} t, \\
G_{3}(v, \eta) & :=\int_{\Omega}\left(-v(x, T) \eta(x, T)-\frac{1}{2}|\eta(x, T)|^{2}\right) \mathrm{d} x, \\
F_{f u_{0}}(\eta) & :=\int_{Q_{T}} f \eta \mathrm{d} x \mathrm{~d} t+\int_{\Omega} u_{0} \eta(x, 0) \mathrm{d} x .
\end{aligned}
$$

Proof: It is not difficult to see that

$$
\begin{gathered}
\mathcal{M}(e):=\sup _{\eta \in \tilde{V}_{0}}\left\{\int_{Q_{T}}\left(\nabla \eta \cdot(\varepsilon \nabla e-\mathbf{F} e)-\frac{\varepsilon}{2}|\nabla \eta|^{2}-\eta_{t} e-\frac{1}{2}\left|\eta_{t}\right|^{2}\right) \mathrm{d} x \mathrm{~d} t+\int_{\Omega}\left(e(x, T) \eta(x, T)-\frac{1}{2}|\eta(x, T)|^{2}\right) \mathrm{d} x\right\} \\
\leq \sup _{\boldsymbol{\tau} \in\left[L_{2}\left(Q_{T}\right)\right]^{d}}\left\{\int_{Q_{T}}\left(\tau \cdot(\varepsilon \nabla e-\mathbf{F} e)-\frac{\varepsilon}{2}|\tau|^{2}\right) \mathrm{d} x \mathrm{~d} t\right\}+\sup _{\xi \in \tilde{V}_{0}}\left\{\int_{Q_{T}}\left(-\xi e-\frac{1}{2}|\xi|^{2}\right) \mathrm{d} x \mathrm{~d} t\right\} \\
\quad+\sup _{\eta(\cdot, T) \in V_{0}}\left\{\int_{\Omega}\left(e(x, T) \eta(x, T)-\frac{1}{2}|\eta(x, T)|^{2}\right) \mathrm{d} x\right\} .
\end{gathered}
$$

Since

$$
\begin{aligned}
\sup _{\boldsymbol{\tau} \in\left[L_{2}\left(Q_{T}\right)\right]^{d}}\left\{\int_{Q_{T}}\left(\tau \cdot(\varepsilon \nabla e-\mathbf{F} e)-\frac{\varepsilon}{2}|\tau|^{2}\right) \mathrm{d} x \mathrm{~d} t\right\} & \leq \frac{1}{2 \varepsilon}\|\varepsilon \nabla e-\mathbf{F} e\|_{Q_{T}}^{2}, \\
\sup _{\xi \in \tilde{V}_{0}}\left\{\int_{Q_{T}}\left(-\xi e-\frac{1}{2}|\xi|^{2}\right) \mathrm{d} x \mathrm{~d} t\right\} & \leq \frac{1}{2}\|e\|_{Q_{T}}^{2}, \\
\sup _{\eta(\cdot, T) \in V_{0}}\left\{\int_{\Omega}\left(e(x, T) \eta(x, T)-\frac{1}{2}|\eta(x, T)|^{2}\right) \mathrm{d} x\right\} & \leq \frac{1}{2}\|e(x, T)\|_{\Omega}^{2},
\end{aligned}
$$

we find that

$$
\begin{aligned}
\mathcal{M}(e) & \leq \frac{1}{2 \varepsilon}\|\varepsilon \nabla e-\mathbf{F} e\|_{Q_{T}}^{2}+\frac{1}{2}\|e\|_{Q_{T}}^{2}+\frac{1}{2}\|e(x, T)\|_{\Omega}^{2} \\
& =\frac{1}{2}\|\nabla e\|_{\varepsilon, Q_{T}}^{2}+\frac{1}{2 \varepsilon}\|\mathbf{F} e\|_{Q_{T}}^{2}+\frac{1}{2}\left\|(\operatorname{div} \mathbf{F})^{1 / 2} e\right\|_{Q_{T}}^{2}+\frac{1}{2}\left\|(-\mathbf{F} \cdot \mathbf{n})^{1 / 2} e\right\|_{S_{T}}^{2}+\frac{1}{2}\|e\|_{Q_{T}}^{2}+\frac{1}{2}\|e(x, T)\|_{\Omega}^{2} \\
& =\frac{1}{2}\|\nabla e\|_{\varepsilon, Q_{T}}^{2}+\frac{1}{2}\left\|\left(\frac{|\mathbf{F}|}{\varepsilon}+\operatorname{div} \mathbf{F}+1\right)^{1 / 2} e\right\|_{Q_{T}}^{2}+\frac{1}{2}\left\|(-\mathbf{F} \cdot \mathbf{n})^{1 / 2} e\right\|_{S_{T}}^{2}+\frac{1}{2}\|e(x, T)\|_{\Omega}^{2},
\end{aligned}
$$

which is exactly defined as $[e]_{\underline{\mathrm{M}}, Q_{T}}^{2}$ in $[59$. On the other hand, by using $[46]$, we see that for any $\eta$ the functional

$$
\mathcal{M}(e)=\sup _{\eta \in \tilde{V}_{0}}\left\{\sum_{i=1}^{3} G_{i}(v, \eta)+F_{f u_{0}}(\eta)\right\}
$$

generates a lower bound for the norm $[e]_{\underline{\mathrm{M}}, Q_{T}}^{2}$. Thus, we arrive at $[59$.

Remark 2 In 62 , the term $\frac{1}{2}\|e\|_{Q_{T}}^{2}$ can be combined with $\frac{1}{2}\|\nabla e\|_{\varepsilon, Q_{T}}^{2}$ by means of Friedrichs' inequality, see (9).

Remark 3 Since the majorants and minorants are equal to norms, see (35), (41), (47) together with (58), and (59), they are nonnegative. In [15], positivity for the solution in the static as well as time-dependent case is proved under the condition that $\boldsymbol{F} \in C^{1}\left(\Omega, \mathbb{R}^{2}\right)$ and $\boldsymbol{F} \cdot \boldsymbol{n}<0$ on $\Gamma_{N}$ or $\boldsymbol{F} \cdot \boldsymbol{n}<0$ on $\Gamma$ in the case of mixed or Robin $B C s$, respectively. For the time-dependent case, the nonnegativity is provided for given nonnegative initial values $u_{0}$. Existence of a minimizing sequence for the majorant as well as a maximizing sequence for the minorant, see for 
instance [33], and corresponding infimum of the majorant and the supremum of the minorant, leads to the estimates of the accuracy of the approximation w.r.t. exact solution. However, it does not automatically imply positivity (or non-negativity). Together with the results on the existence of a minimizing sequence for the majorant as well as a maximizing sequence for the minorant, see for instance [41, Section 3.6], the infimum of the majorant and the supremum of the minorant both lead to the exact (nonnegative) solution.

Remark 4 Note that the model problems $(12)-(13)$ and $\sqrt{19}-(22)$ are written in form of a first-order system of conservation laws supplied by a proper constitutive relation for the flux. This could yield to the extension of this work's functional-based error analysis to the use of a dual mixed weak formulation of the PDE system, which would be of great interest in the implementation of computations for more realistic applications, where the approximation of the total flux $(\mathbf{F} u-\boldsymbol{p})$ is often more important than the approximation of $u$ itself. Studies concerning the functionalbased error analysis devoted to a posteriori error estimates of mixed approximations for the Poisson equation with Dirichlet and mixed Dirichlet/Neumann boundary conditions can be found in [42] and [43], respectively. Moreover, in the latter work it is shown that after an appropriate scaling of the coordinates and the equation, the ratio of the upper and lower bounds for the error in the product norm never exceeds the constant 3. Such an a posteriori error approach is computationally very cheap and can also be used for the indication of the local error distribution. As an application, [43] considers the linear elasticity problem, where the reconstruction of the stresses (dual variable) is equally important to the reconstruction of the displacement (primal variable).

\section{$5 \quad$ Numerical examples}

We have presented a generalized error control method, namely, the functional error estimates, for convectiondominated diffusion problems in Sections 3 and 4 . They are derived independently from the numerical method used to reconstruct the approximation. As a result, one does not need to adapt the obtained error estimates to the stabilization technique chosen in the solver but can just use the reconstructed approximation.

The current section presents numerical results for the problems $\sqrt{12})-(13)$ and $(19)-(22)$ in the static and timedependent case, respectively. The examples were computed for various parameters and boundary conditions. A particularly interesting setting (discussed throughout this section) is the convection-dominated problem, namely, when $\varepsilon \ll\|\mathbf{F}\|_{L^{\infty}}$. In that case, the solution contains a boundary layer, which can be either of the width $O(\varepsilon)$ (the so-called regular boundary layer) or of the width $O(\sqrt{\varepsilon})$ (parabolic boundary layer). The detailed discussion addressing properties for both of these layers can be found in [50].

The stabilised form of 24 can be written as follows:

$$
a(u, \eta)+\sum_{K \in \mathcal{K}_{h}} \delta_{K} s_{K}(u, \eta)=\langle f, \eta\rangle_{\Omega},
$$

where $s_{K}(u, \eta)$ depends on the approach used and $\delta_{K}$ is a non-negative stabilisation parameter, which can vary from one method to another. In this work, we use the Streamline Upwind Petrov-Galerkin (SUPG) introduced by Brooks and Hughes [12, where

$$
s_{K}(u, v)=\int_{K}(-\Delta u+\nabla(\mathbf{F} u), \mathbf{F} \cdot \nabla u) .
$$

The stabilisation parameter $\delta_{K}$ is defined depending on the cell's Peclét number, i.e.,

$$
P_{K}:=\frac{\|\mathbf{F}\|_{\infty, K} h_{K}}{2 \varepsilon},
$$

where $\|\cdot\|_{\infty, K}$ is the norm in $\left[L^{\infty}(K)\right]^{d}, h_{K}$ denotes the diameter of a finite element $K$, and $\mathbf{F}$ prescribes the direction of the convective flow. Then,

$$
\delta_{K}:= \begin{cases}\delta_{0} \frac{h_{K}}{\|\mathbf{F}\|_{\infty}, K}, & \text { if } \quad P_{K}>1 \quad \text { (convection-dominated case) } \\ \delta_{1} \frac{h_{K}^{2}}{\varepsilon}, & \text { if } \quad P_{K} \leq 1 \quad \text { (diffusion-dominated case) }\end{cases}
$$

where $\delta_{0}, \delta_{1}>0$ are appropriately chosen constants. Set $\delta_{K}:=\frac{h_{K}}{2|\mathbf{F}|}$, where $|\mathbf{F}|$ stands for the magnitude of the vector. All the numerical results obtained in the section are carried out in Python and $\mathrm{C}++$ in the framework of The FEniCS Project [52, 32. 


\begin{tabular}{|c|c|c|c|c|c|c|c|c|c|c|c|c|}
\hline & \multicolumn{6}{|c|}{$v \in \mathrm{P}_{1}$} & \multicolumn{6}{|c|}{$v \in \mathrm{P}_{2}$} \\
\hline ref. \# & {$[e]_{\overline{\mathrm{M}}}$} & $\begin{array}{l}\text { e.o.c. } \\
\left([e]_{\overline{\mathrm{M}}}\right)\end{array}$ & $\overline{\mathrm{M}}$ & $\begin{array}{c}\text { e.o.c. } \\
(\overline{\mathrm{M}})\end{array}$ & $\underline{M}$ & $\begin{array}{c}\text { e.o.c. } \\
(\underline{\mathrm{M}})\end{array}$ & {$[e]_{\overline{\mathrm{M}}}$} & $\begin{array}{l}\text { e.o.c. } \\
\left([e]_{\bar{M}}\right)\end{array}$ & $\overline{\mathrm{M}}$ & $\begin{array}{c}\text { e.o.c. } \\
(\overline{\mathrm{M}})\end{array}$ & $\underline{M}$ & $\begin{array}{l}\text { e.o.c. } \\
(\underline{\mathrm{M}})\end{array}$ \\
\hline 5 & $5.66 \mathrm{e}-01$ & 0.31 & $1.29 \mathrm{e}+00$ & 0.98 & $1.06 \mathrm{e}+00$ & 0.85 & $2.71 \mathrm{e}-01$ & 0.75 & $5.15 \mathrm{e}-01$ & 1.27 & $4.54 \mathrm{e}-01$ & 1.22 \\
\hline 7 & $2.97 \mathrm{e}-01$ & 0.92 & $3.24 \mathrm{e}-01$ & 1.01 & $3.19 \mathrm{e}-01$ & 1.00 & $5.64 \mathrm{e}-02$ & 1.86 & $5.90 \mathrm{e}-02$ & 1.90 & $5.86 \mathrm{e}-02$ & 1.90 \\
\hline 9 & $7.94 \mathrm{e}-02$ & 0.99 & $7.99 \mathrm{e}-02$ & 1.00 & $7.97 \mathrm{e}-02$ & 1.00 & $3.99 \mathrm{e}-03$ & 1.99 & $4.01 \mathrm{e}-03$ & 1.99 & $4.00 \mathrm{e}-03$ & 1.99 \\
\hline 11 & $1.99 \mathrm{e}-02$ & 1.00 & $1.99 \mathrm{e}-02$ & 1.00 & $1.99 \mathrm{e}-02$ & 1.00 & $2.51 \mathrm{e}-04$ & 2.00 & $2.51 \mathrm{e}-04$ & 2.00 & $2.51 \mathrm{e}-04$ & 2.00 \\
\hline
\end{tabular}

Table 1: Example 1. Error order of convergence (e.o.c.) for approximations $v \in \mathrm{P}_{1}$ and $\boldsymbol{y} \in \mathrm{RT}_{1}$ as well as $v \in \mathrm{P}_{2}$ and $\boldsymbol{y} \in \mathrm{RT}_{1}$ w.r.t. refinement steps.

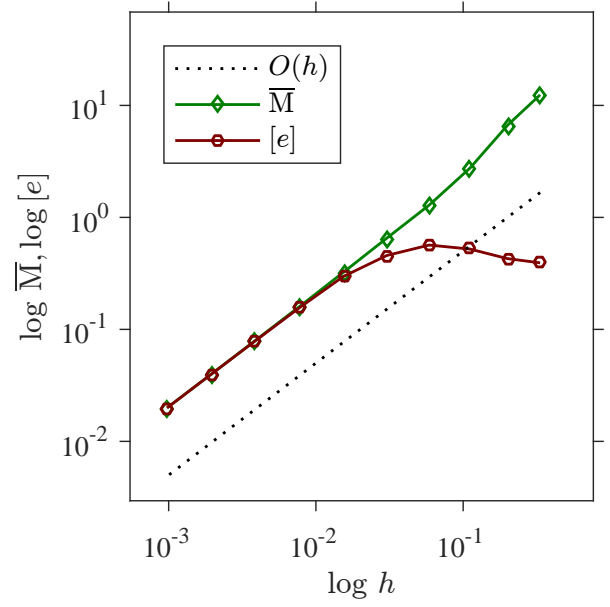

(a) $v \in \mathrm{P}_{1}$

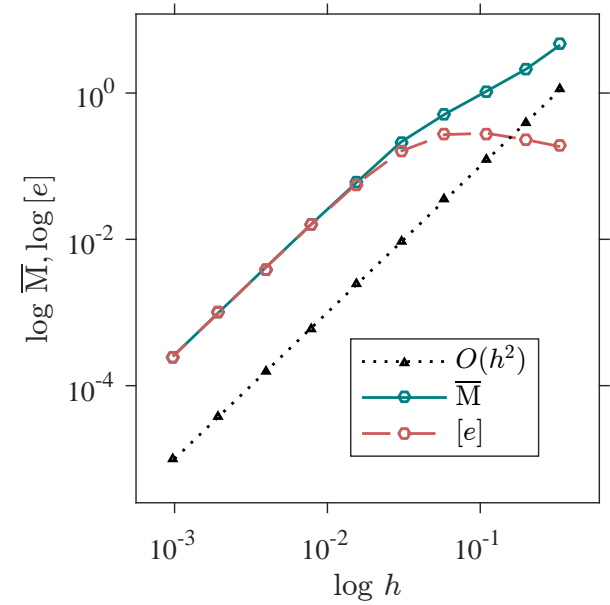

(b) $v \in \mathrm{P}_{2}$

Figure 1: Example 1. Error order of convergence (e.o.c.) for (a) $v \in \mathrm{P}_{1}$ and $\boldsymbol{y} \in \mathrm{RT}_{1}$ as well as (b) $v \in \mathrm{P}_{2}$ and $\boldsymbol{y} \in \mathrm{RT}_{1}$ w.r.t. degrees of freedom (d.o.f.).

Example 1 Consider the one-dimensional classical example on the unit interval $\Omega=(0,1)$ :

$$
-\varepsilon u_{x x}+a u_{x}=0, \quad u(0)=u(1)=0,
$$

with the solution $u=\frac{1-e^{a x / \varepsilon}}{-e^{a / \varepsilon}}$ known to have boundary layer in the neighborhood of the right part of the boundary $x=1$. We select $a=1.0$ and $\varepsilon=1 e-2$. Its approximation $v$ is reconstructed for two cases with Lagrangian finite elements (FEs) of order $1\left(v \in \mathrm{P}_{1}\right)$ and of order $2\left(v \in \mathrm{P}_{2}\right)$. The correctness of the numerical solver is confirmed by the error order of convergence (e.o.c.) for the uniform refinement procedure executed for both $v \in \mathrm{P}_{1}$ and $v \in \mathrm{P}_{2}$ (see Table 1). The same results are illustrated in Figure 1 In both cases, the initial mesh contains of 8 elements and 9 nodes, and we proceed for 12 global refinement steps. As expected, the error behaves as $O(h)$ and $O\left(h^{2}\right)$ in the first and the second case, respectively. Here, the flux $\boldsymbol{y}$ is reconstructed with Raviart-Thomas elements of the first order denoted by $\mathrm{RT}_{1}$ as follows

$$
R T_{1}\left(\mathcal{K}_{h}\right):=\left\{\boldsymbol{y} \in H(\operatorname{div}, K): \forall K \in \mathcal{K}_{h} \quad \exists \boldsymbol{q} \in\left(\mathrm{P}_{1}\right)^{d}, r \in \mathrm{P}_{1} \quad \forall \boldsymbol{x} \in K: \boldsymbol{y}(\boldsymbol{x})=\boldsymbol{q}(\boldsymbol{x})+\boldsymbol{x} r(\boldsymbol{x})\right\} .
$$

The auxiliary function $w$ for the minorant reconstruction is approximated by $\mathrm{P}_{3}$-Lagrangian FEs. The values of the majorants and minorants as well as their efficiency indices w.r.t. several refinement steps are presented in Table 1. It is easy to see that both upper and lower bounds stay rather sharp w.r.t. the error measures $[e]_{\overline{\mathrm{M}}}$ and $[e]_{\underline{\mathrm{M}}}$, respectively.

Example 2 Next, we consider the two-dimensional example defined on the unit square domain $\Omega=(0,1)^{2}$ :

$$
-\varepsilon \Delta u+\mathbf{F} \cdot \nabla u=x, \quad u=0 \quad \text { on } \quad \partial \Omega,
$$

where $\varepsilon=1 e-3$ and $\mathbf{F}=(-x, 0)^{\mathrm{T}}$. In this case, the exact solution is not known. However, by following the analysis presented in [47, one can predict the location of the boundary layer. The approximation $v$ is reconstructed by 


\begin{tabular}{c|c|ccc|ccc} 
ref. \# & d.o.f. \# & {$[e]_{\overline{\mathrm{M}}}$} & $\overline{\mathrm{M}}$ & $I_{\text {eff }}(\overline{\mathrm{M}})$ & {$[e]_{\underline{\mathrm{M}}}$} & $\underline{\mathrm{M}}$ & $I_{\text {eff }}(\underline{\mathrm{M}})$ \\
\hline 0 & 9 & $8.08 \mathrm{e}-01$ & $8.45 \mathrm{e}+00$ & 10.46 & $2.81 \mathrm{e}+00$ & $2.75 \mathrm{e}+00$ & 0.98 \\
2 & 33 & $5.09 \mathrm{e}-01$ & $1.31 \mathrm{e}+00$ & 2.56 & $6.36 \mathrm{e}-01$ & $6.38 \mathrm{e}-01$ & 1.00 \\
4 & 129 & $1.57 \mathrm{e}-01$ & $2.50 \mathrm{e}-01$ & 1.59 & $1.59 \mathrm{e}-01$ & $1.59 \mathrm{e}-01$ & 1.00 \\
6 & 513 & $3.98 \mathrm{e}-02$ & $5.21 \mathrm{e}-02$ & 1.31 & $3.99 \mathrm{e}-02$ & $3.99 \mathrm{e}-02$ & 1.00 \\
8 & 2049 & $9.97 \mathrm{e}-03$ & $1.15 \mathrm{e}-02$ & 1.16 & $9.97 \mathrm{e}-03$ & $9.97 \mathrm{e}-03$ & 1.00 \\
10 & 8193 & $2.49 \mathrm{e}-03$ & $2.68 \mathrm{e}-03$ & 1.07 & $2.49 \mathrm{e}-03$ & $2.49 \mathrm{e}-03$ & 1.00 \\
12 & 32769 & $6.23 \mathrm{e}-04$ & $6.44 \mathrm{e}-04$ & 1.03 & $6.23 \mathrm{e}-04$ & $6.23 \mathrm{e}-04$ & 1.00
\end{tabular}

Table 2: Example 1. Majorant, minorant, and corresponding efficiency indices.

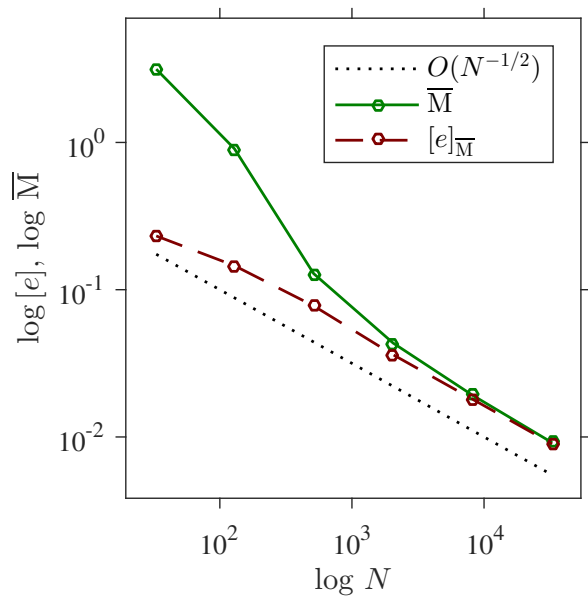

(a) $[e]_{\overline{\mathrm{M}}}$ and $\overline{\mathrm{M}}$

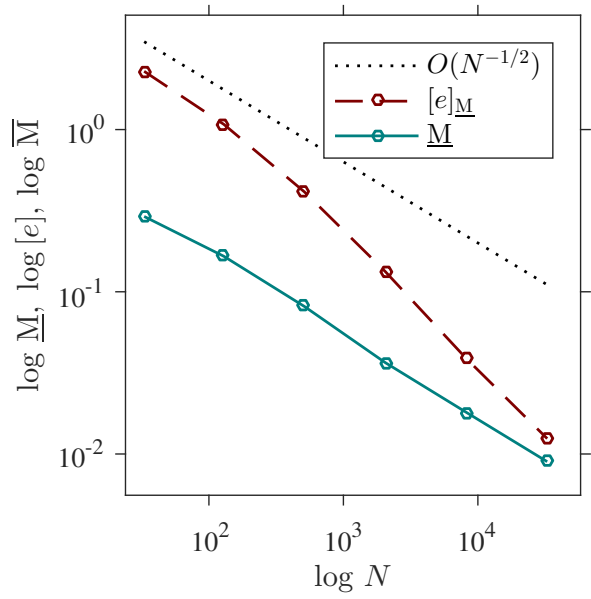

(b) $[e]_{\mathrm{M}}$ and $\underline{\mathrm{M}}$

Figure 2: Example 2. Convergence of (a) $[e]_{\overline{\mathrm{M}}}$ and $\overline{\mathrm{M}}$ as well as (b) $[e]_{\underline{\mathrm{M}}}$ and $\underline{\mathrm{M}}$.

\begin{tabular}{c|c|ccc|ccc} 
ref. \# & d.o.f. \# & {$[e]_{\overline{\mathrm{M}}}$} & $\overline{\mathrm{M}}$ & $I_{\mathrm{eff}}(\overline{\mathrm{M}})$ & {$[e]_{\underline{\mathrm{M}}}$} & $\underline{\mathrm{M}}$ & $I_{\text {eff }}(\underline{\mathrm{M}})$ \\
\hline 0 & 25 & $2.32 \mathrm{e}-01$ & $3.15 \mathrm{e}+00$ & 13.57 & $2.29 \mathrm{e}+00$ & $2.91 \mathrm{e}-01$ & 0.13 \\
2 & 289 & $7.77 \mathrm{e}-02$ & $1.29 \mathrm{e}-01$ & 1.66 & $4.13 \mathrm{e}-01$ & $8.12 \mathrm{e}-02$ & 0.20 \\
4 & 4225 & $1.80 \mathrm{e}-02$ & $1.92 \mathrm{e}-02$ & 1.06 & $3.88 \mathrm{e}-02$ & $1.81 \mathrm{e}-02$ & 0.47 \\
6 & 66049 & $4.47 \mathrm{e}-03$ & $4.49 \mathrm{e}-03$ & 1.01 & $4.95 \mathrm{e}-03$ & $4.46 \mathrm{e}-03$ & 0.90
\end{tabular}

Table 3: Example 2, Majorant, minorant, and corresponding efficiency indices.

$\mathrm{P}_{1}$-elements, whereas the majorant is reconstructed with an auxiliary function $\boldsymbol{y} \in \mathrm{RT}_{1}$ and the minorant with an auxiliary function $w \in \mathrm{P}_{3}$.

Let us first consider the numerical experiments obtained by globally refining the mesh. The order of convergence of $\underline{\mathrm{M}}$ and $\overline{\mathrm{M}}$ is confirmed in Figure 2. Due to their high quantitative efficiency, the lines of the error estimates and corresponding errors almost coincide on the plots. Table 3 illustrates values of both minorant and majorant as well as their efficiency indices w.r.t. the error measures that they bound.

Next, we test the performance of an adaptive strategy of the refinement. It is based on the local distribution of the indicator, following from the majorant functional. We choose a widely-used bulk marking criterion for selecting elements to be refined denoted by $\operatorname{IM}_{\text {BULK }}(\theta)$, see $[18$. Here, the bulk parameter is set to $\theta=0.3$. Table 5 provides the values for the efficiency indices of the majorant and minorant, which are getting sharper as the refinement steps proceed. The evolution of the meshes is illustrated in Figure 3. Here, the left column presents the meshes constructed on refinement steps 3-4, whereas the right column illustrates the distribution of the local error values on the elements of the mesh. The elements with the highest and lowest errors are indicated by the range of colours between red and blue, respectively. From the meshes, one can also see that refinement based on the majorant resolves the boundary layers at $x=0$.

Example 3 Next, we consider yet another convection-dominated example on the unit square $\Omega=(0,1)^{2}$ :

$$
-\operatorname{div}(A \nabla u)+\nabla(\mathbf{F} u)=f, \quad u=0 \quad \text { on } \quad \partial \Omega,
$$




\begin{tabular}{c|c|ccc|ccc} 
ref. \# & d.o.f. \# & {$[e]_{\overline{\mathrm{M}}}$} & $\overline{\mathrm{M}}$ & $I_{\text {eff }}(\overline{\mathrm{M}})$ & {$[e]_{\underline{\mathrm{M}}}$} & $\underline{\mathrm{M}}$ & $I_{\text {eff }}(\underline{\mathrm{M}})$ \\
\hline 0 & 25 & $4.06 \mathrm{e}-02$ & $4.66 \mathrm{e}-02$ & 1.15 & $4.06 \mathrm{e}-02$ & $4.06 \mathrm{e}-02$ & 1.00 \\
2 & 109 & $1.50 \mathrm{e}-02$ & $1.56 \mathrm{e}-02$ & 1.04 & $1.50 \mathrm{e}-02$ & $1.50 \mathrm{e}-02$ & 1.00 \\
4 & 576 & $6.53 \mathrm{e}-03$ & $6.66 \mathrm{e}-03$ & 1.02 & $6.53 \mathrm{e}-03$ & $6.53 \mathrm{e}-03$ & 1.00 \\
6 & 2870 & $2.99 \mathrm{e}-03$ & $3.02 \mathrm{e}-03$ & 1.01 & $2.99 \mathrm{e}-03$ & $2.99 \mathrm{e}-03$ & 1.00 \\
8 & 14527 & $1.35 \mathrm{e}-03$ & $1.35 \mathrm{e}-03$ & 1.00 & $1.35 \mathrm{e}-03$ & $1.35 \mathrm{e}-03$ & 1.00
\end{tabular}

Table 4: Example 2. Majorant, minorant, and corresponding efficiency indices for an adaptive refinement strategy.

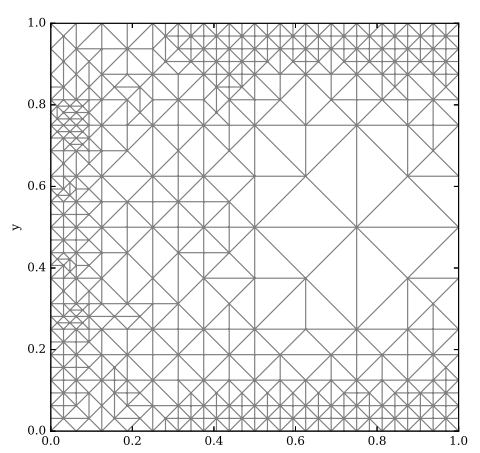

ref. \# 3:

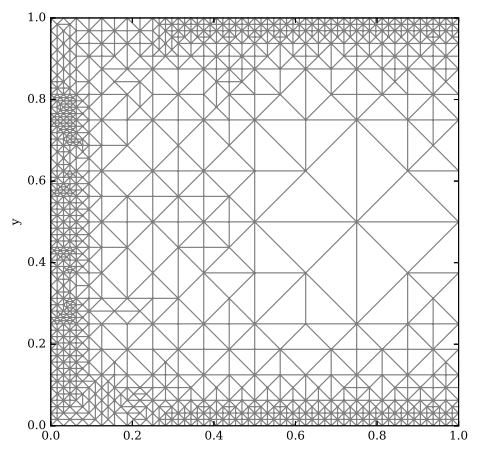

ref. \# 4:

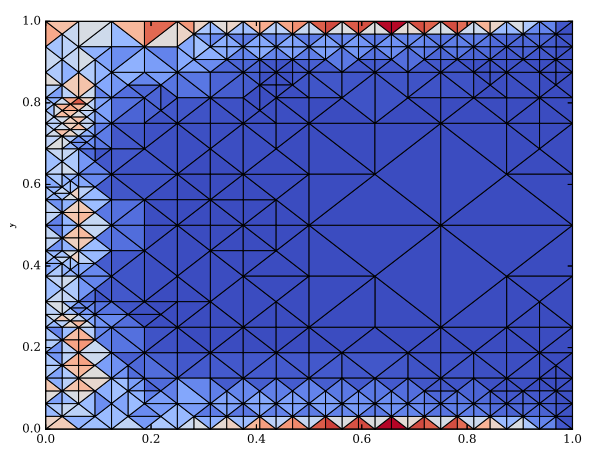

ref. \# 3:

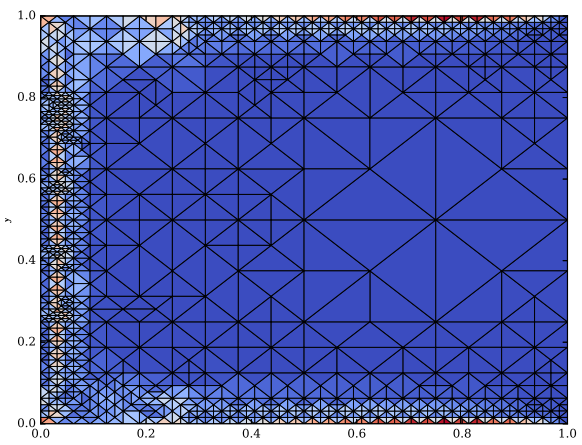

ref. \# 4:

Figure 3: Example 2, Meshes generated by the adaptive refinement strategy and the distribution of the local error (right column) with $\mathbb{M}_{\mathrm{BULK}}(0.3)$.

\begin{tabular}{c|c|ccc|ccc} 
ref. \# & d.o.f. \# & {$[e]_{\overline{\mathrm{M}}}$} & $\overline{\mathrm{M}}$ & $I_{\text {eff }}(\overline{\mathrm{M}})$ & {$[e]_{\underline{\mathrm{M}}}$} & $\underline{\mathrm{M}}$ & $I_{\text {eff }}(\underline{\mathrm{M}})$ \\
\hline 0 & 25 & $2.32 \mathrm{e}-01$ & $3.15 \mathrm{e}+00$ & 13.57 & $2.29 \mathrm{e}+00$ & $2.91 \mathrm{e}-01$ & 0.13 \\
2 & 101 & $1.18 \mathrm{e}-01$ & $4.47 \mathrm{e}-01$ & 3.80 & $9.98 \mathrm{e}-01$ & $1.31 \mathrm{e}-01$ & 0.13 \\
4 & 469 & $4.05 \mathrm{e}-02$ & $5.19 \mathrm{e}-02$ & 1.28 & $1.41 \mathrm{e}-01$ & $4.11 \mathrm{e}-02$ & 0.29 \\
6 & 2319 & $1.27 \mathrm{e}-02$ & $1.36 \mathrm{e}-02$ & 1.07 & $1.95 \mathrm{e}-02$ & $1.27 \mathrm{e}-02$ & 0.65 \\
8 & 11753 & $5.43 \mathrm{e}-03$ & $5.51 \mathrm{e}-03$ & 1.02 & $6.04 \mathrm{e}-03$ & $5.43 \mathrm{e}-03$ & 0.90
\end{tabular}

Table 5: Example 3. Majorant, minorant, and corresponding efficiency indices for an adaptive refinement strategy.

where $A=\left[\begin{array}{cc}10 & 0 \\ 0 & 1\end{array}\right], f=x+y$, and $\mathbf{F}=\left(-\cos \left(\frac{\pi}{3}\right) x, \sin \left(\frac{\pi}{3}\right) y\right)^{\mathrm{T}}$. Again, let the approximation $v$ be reconstructed with $\mathrm{P}_{1}$-elements, $\boldsymbol{y}$ by $\mathrm{RT}_{1}$-elements, and $w \in \mathrm{P}_{3}$ (the optimal error convergence is presented in Figure $4 \mathrm{~b}$ ). The approximate solution obtained with an adaptive refinement is illustrated in Figure $4 \mathrm{a}$, where one can see that the solution convects towards the boundary $y=1$.

By the current example, we aim to show that even for the anisotropic $A$ and heterogeneous $\mathbf{F}$ the performance of the error estimates remains rather sharp. In particular, Table 5 confirms that the efficiency indices of the minorant and the majorant lie close to 1 as the refinements evolve. 


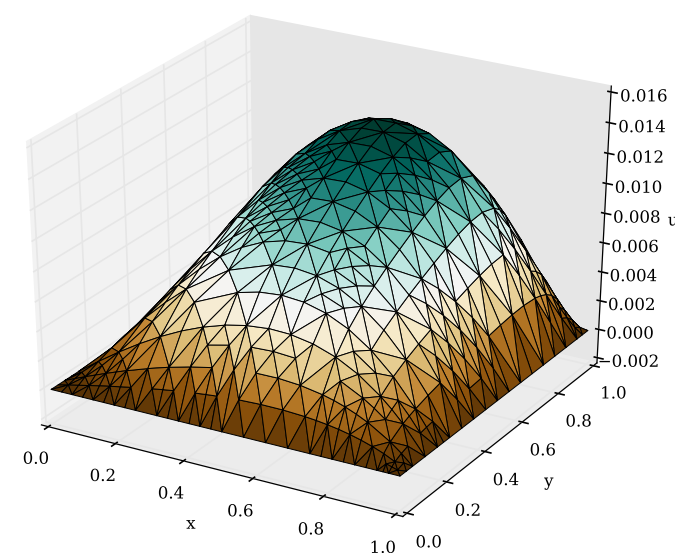

(a) $v$, adaptive ref. \# 6

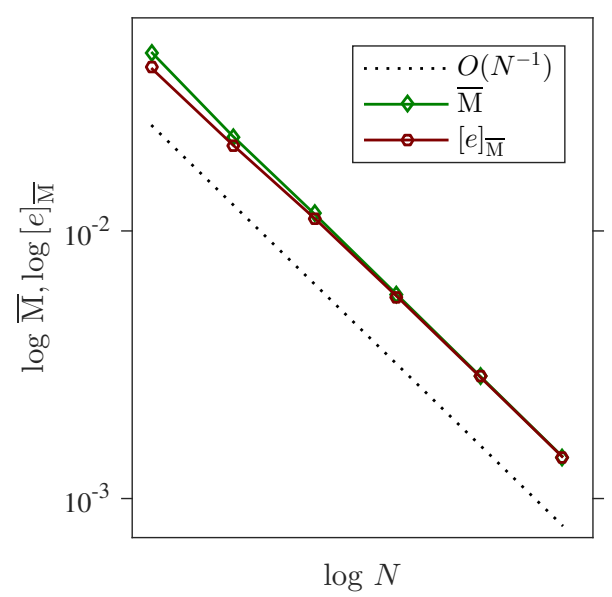

(b) $v$, adaptive ref. \# 6

Figure 4: Example 3. (a) Approximate solution on the adaptive refinement step \# 4. (b) Optimal order of convergence together with error and majorant order of convergence.

\begin{tabular}{|c|c|c|c|c|c|c|}
\hline$N^{-1 / d}$ & {$[e]$} & e.o.c. $([e])$ & $\overline{\mathrm{M}}$ & e.o.c. $(\overline{\mathrm{M}})$ & $\underline{\mathrm{M}}$ & e.o.c. $(\underline{\mathrm{M}})$ \\
\hline \multicolumn{7}{|c|}{$\theta=0.3$} \\
\hline 191 & $7.66 \mathrm{e}-02$ & 4.57 & $5.26 \mathrm{e}-01$ & 1.17 & $4.83 \mathrm{e}-01$ & 1.05 \\
\hline 808 & $1.08 \mathrm{e}-01$ & 2.27 & $2.17 \mathrm{e}-01$ & 1.44 & $2.13 \mathrm{e}-01$ & 1.41 \\
\hline 3478 & $6.01 \mathrm{e}-02$ & 1.93 & $6.96 \mathrm{e}-02$ & 1.88 & $6.91 \mathrm{e}-02$ & 1.86 \\
\hline 15724 & $1.84 \mathrm{e}-02$ & 1.66 & $1.86 \mathrm{e}-02$ & 1.63 & $1.85 \mathrm{e}-02$ & 1.65 \\
\hline 32918 & $1.01 \mathrm{e}-02$ & 1.03 & $1.02 \mathrm{e}-02$ & 0.98 & $1.01 \mathrm{e}-02$ & 1.02 \\
\hline 74789 & $6.60 \mathrm{e}-03$ & 0.92 & $6.79 \mathrm{e}-03$ & 0.78 & $6.63 \mathrm{e}-03$ & 0.91 \\
\hline \multicolumn{7}{|c|}{$\theta=0.4$} \\
\hline 244 & $7.65 \mathrm{e}-02$ & 3.86 & $5.26 \mathrm{e}-01$ & 1.01 & $4.84 \mathrm{e}-01$ & 0.89 \\
\hline 1290 & $1.08 \mathrm{e}-01$ & 1.95 & $2.15 \mathrm{e}-01$ & 1.24 & $2.12 \mathrm{e}-01$ & 1.22 \\
\hline 7056 & $5.99 \mathrm{e}-02$ & 1.62 & $6.91 \mathrm{e}-02$ & 1.58 & $6.86 \mathrm{e}-02$ & 1.57 \\
\hline 16822 & $3.39 \mathrm{e}-02$ & 1.37 & $3.47 \mathrm{e}-02$ & 1.35 & $3.47 \mathrm{e}-02$ & 1.36 \\
\hline
\end{tabular}

Table 6: Example 4 Error order of convergence for approximations $v \in \mathrm{P}_{1}$ and $\boldsymbol{y} \in \mathrm{RT}_{1}$ for bulk parameters $\theta=0.3$ and $\theta=0.4$.

\subsection{Static convection-dominated problems}

Next, we discuss the following set of example problems: consider the reaction-convection-diffusion equation

$$
-\varepsilon \Delta u+\mathbf{a} \cdot \nabla u+\lambda u=0
$$

(which basically yields from (12)- 13 ) with homogeneous Dirichlet BCs. However, in contrast to the previous examples, we assume now that reaction and convection are independent of each other.

Example 4 Assume that $d=2, \mathbf{a}=(1,0)^{\mathrm{T}}, \varepsilon=1 e-3, \lambda=1$, and the exact solution is defined by the function $u=\left(x+\frac{e^{(x-1) / \varepsilon}-e^{-1 / \varepsilon}}{e^{-1 / \varepsilon}-1}\right) y(1-y)$ with a boundary layer in the neighbourhood of $x=1$. Its approximation $v$ is reconstructed by $\mathrm{P}_{1}$ elements (see Figure 5a). Since the uniform refinement does not provide expected orders of convergence, we apply an adaptive refinement with bulk marking criterion $\operatorname{IM}_{\mathrm{BULK}}(\theta)$. We choose two different parameters, i.e., $\theta=0.3$ and $\theta=0.4$, to confirm optimal convergence order for $\overline{\mathrm{M}}$ (see Table 6 and Figure $5 \mathrm{~b}$ ). The values of the total majorants and minorants as well as corresponding efficiency indices are presented in Table 7 w.r.t. several refinement steps. It is easy to see that even though the efficiency indices are high on the initial steps, both majorant and minorant become rather sharp towards the 9-th refinement step. The evolution of the meshes corresponding to the different $\theta$ parameters is presented in Figure 6 . 


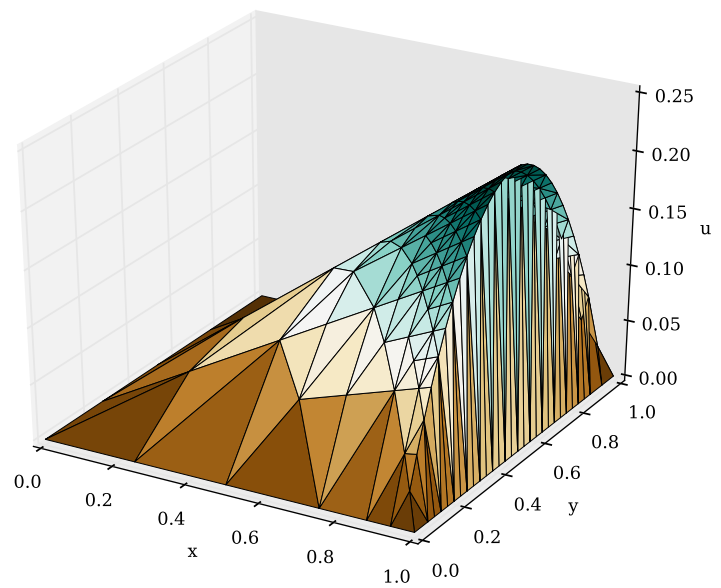

(a) $v \in P^{1}$, ref. \# 5

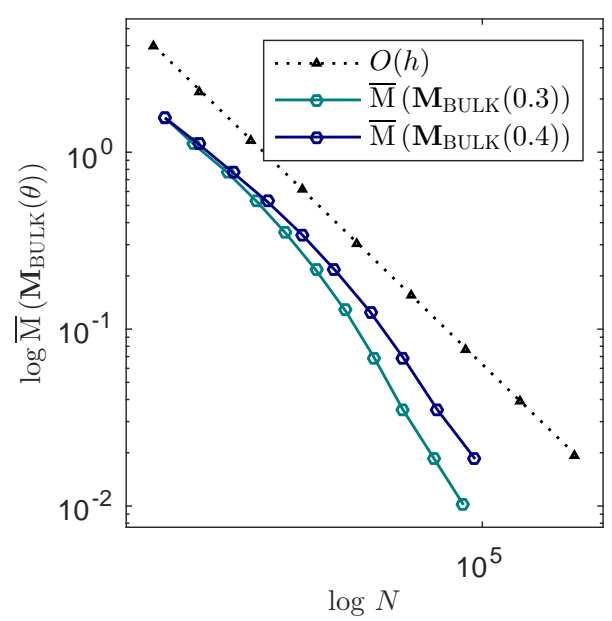

(b) adaptive refinement

Figure 5: Example 4 (a) The approximate solution on the mesh (1089 nodes, 2048 elements). (b) Majorant's order of convergence for bulk parameters $\theta=0.3$ and $\theta=0.4$.

\begin{tabular}{|c|c|c|c|c|c|c|c|}
\hline ref. \# & d.o.f. \# & {$[e]_{\overline{\mathrm{M}}}$} & $\overline{\mathrm{M}}$ & $I_{\mathrm{eff}}(\overline{\mathrm{M}})$ & {$[e]_{\underline{\mathrm{M}}}$} & $\underline{\mathrm{M}}$ & $I_{\mathrm{eff}}(\underline{\mathrm{M}})$ \\
\hline \multicolumn{8}{|c|}{$\theta=0.3$} \\
\hline 1 & 45 & $5.19 \mathrm{e}-02$ & $1.11 \mathrm{e}+00$ & 21.41 & $1.09 \mathrm{e}+00$ & $6.53 \mathrm{e}-01$ & 0.60 \\
\hline 3 & 191 & $7.66 \mathrm{e}-02$ & $5.26 \mathrm{e}-01$ & 6.86 & $5.22 \mathrm{e}-01$ & $4.83 \mathrm{e}-01$ & 0.93 \\
\hline 5 & 808 & $1.08 \mathrm{e}-01$ & $2.17 \mathrm{e}-01$ & 2.02 & $2.20 \mathrm{e}-01$ & $2.13 \mathrm{e}-01$ & 0.97 \\
\hline 7 & 3478 & $6.01 \mathrm{e}-02$ & $6.96 \mathrm{e}-02$ & 1.16 & $7.01 \mathrm{e}-02$ & $6.91 \mathrm{e}-02$ & 0.99 \\
\hline 9 & 15724 & $1.84 \mathrm{e}-02$ & $1.86 \mathrm{e}-02$ & 1.01 & $1.86 \mathrm{e}-02$ & $1.85 \mathrm{e}-02$ & 1.00 \\
\hline 11 & 74789 & $6.60 \mathrm{e}-03$ & $6.79 \mathrm{e}-03$ & 1.03 & $6.71 \mathrm{e}-03$ & $6.63 \mathrm{e}-03$ & 0.99 \\
\hline \multicolumn{8}{|c|}{$\theta=0.4$} \\
\hline 1 & 51 & $5.25 \mathrm{e}-02$ & $1.11 \mathrm{e}+00$ & 21.09 & $1.10 \mathrm{e}+00$ & $6.53 \mathrm{e}-01$ & 0.59 \\
\hline 3 & 244 & $7.65 \mathrm{e}-02$ & $5.26 \mathrm{e}-01$ & 6.87 & $5.20 \mathrm{e}-01$ & $4.84 \mathrm{e}-01$ & 0.93 \\
\hline 5 & 1290 & $1.08 \mathrm{e}-01$ & $2.15 \mathrm{e}-01$ & 2.00 & $2.15 \mathrm{e}-01$ & $2.12 \mathrm{e}-01$ & 0.98 \\
\hline 7 & 7056 & $5.99 \mathrm{e}-02$ & $6.91 \mathrm{e}-02$ & 1.15 & $6.95 \mathrm{e}-02$ & $6.86 \mathrm{e}-02$ & 0.99 \\
\hline 9 & 42827 & $1.83 \mathrm{e}-02$ & $1.85 \mathrm{e}-02$ & 1.01 & $1.84 \mathrm{e}-02$ & $1.84 \mathrm{e}-02$ & 1.00 \\
\hline
\end{tabular}

Table 7: Example 4 Majorant, minorant, and corresponding efficiency indices.

According to the theory of asymptotic expansion [23, the solution of the original problem depends continuously on $\varepsilon$. Using this fact, the authors of [16, 51] use the so-called multilevel-homotopic-adaptive finite element method (MHA FEM) with respect to the diffusion constant $\varepsilon$, such that grids are iteratively adapted to a better approximate solution as the diffusion parameter gets decreased. The method gets initialised with $\tilde{\varepsilon}=\varepsilon_{0}$ (e.g., $\left.\varepsilon_{0}=1\right)$, and the mesh of the ad hoc diffusion problem

$$
-\tilde{\varepsilon} \Delta u+\mathbf{a} \cdot \nabla u+\lambda u=0
$$

gets refined adaptively. On the next iteration, the value of $\tilde{\varepsilon}$ is reduced, and the mesh adaptation is performed for the problem with the updated $\tilde{\varepsilon}$. Iterations continue until the desired value of $\varepsilon$ is reached, i.e., until condition $\tilde{\varepsilon}>\varepsilon$ is satisfied. For the reader's convenience, the steps described above are summarised in Algorithm 11(see also [51). We use the MHA FEM in combination with the streamline diffusion FEM, where mesh adaptation is driven by the local distribution of the error indicator following from the majorant. Such an approach can also serve as a preprocessing for a problem so that one can generate problem-adapted meshes once realistic problems are concerned.

Example 5 Assume now that $d=2, \mathbf{a}=(1,0)^{\mathrm{T}}, \varepsilon=1 e-4, \lambda=0$, and the RHS $f$ as well as and the boundary data $g=0$ are chosen such that the solution of the problem is defined by

$$
u=\left(x^{2}-e^{x-1 / \varepsilon}\right) y(1-y)
$$

(see Figure 7 illustrating the approximate solution on the uniformly refined mesh). It exhibits an exponentially regular boundary (of the width $O(\varepsilon)$ ) near $\{x=1\}$ perpendicular to the convection direction $(1,0)^{\mathrm{T}}$ (see also [51]). 


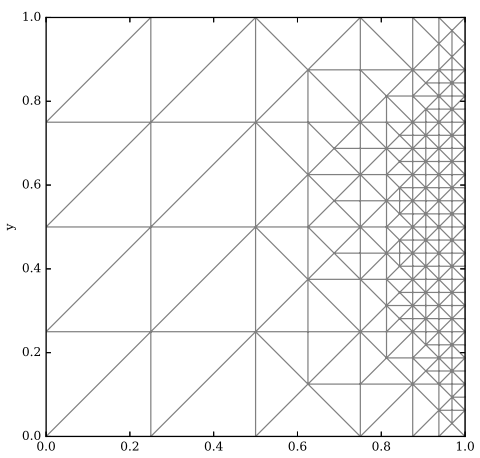

(a) ref. $\# 2, \theta=0.3$

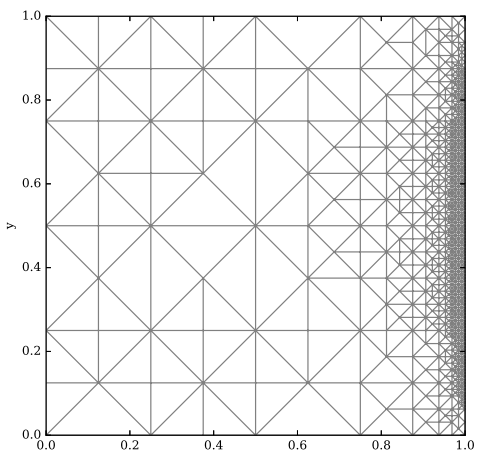

(c) ref. \# $4, \theta=0.3$

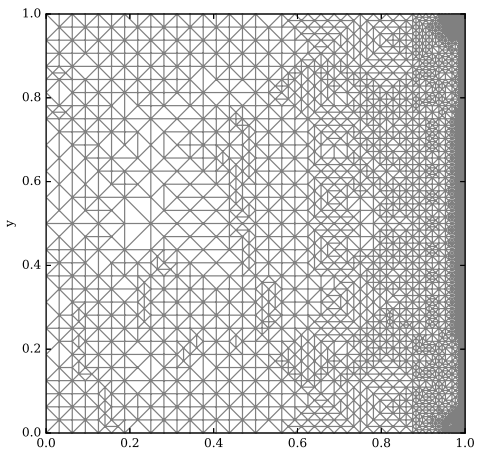

(e) ref. \# 6, $\theta=0.3$

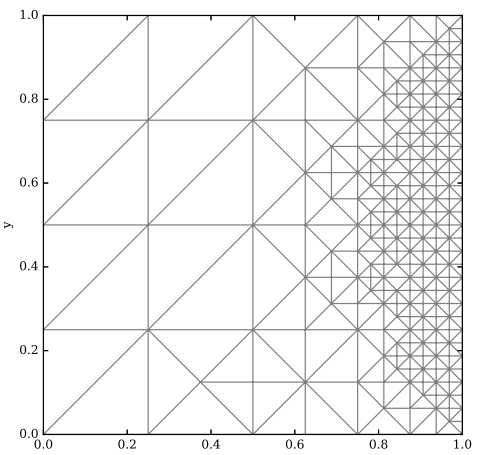

(b) ref. \# 2, $\theta=0.4$

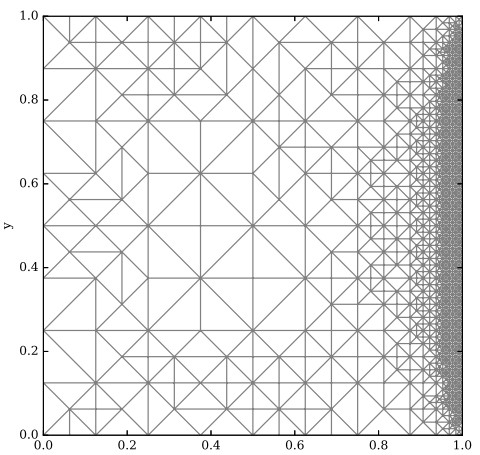

(d) ref. \# $4, \theta=0.4$

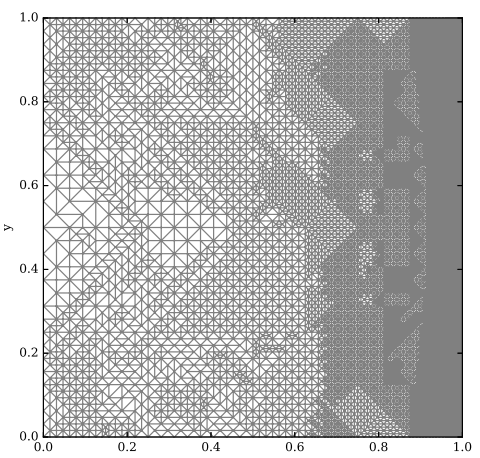

(f) ref. \# 6, $\theta=0.4$

Figure 6: Example 4. Evolution of the mesh on the refinement steps 2, 4, 6 with different $\theta=0.3$ (left column) and $\theta=0.4$ (right column).

We test two different scenarios of updating the parameter $\varepsilon$. The first one contains 4 adaptive refinement steps for each of the levels $\varepsilon$, and the diffusion parameter decreases by 10 on each level. Table 8 contains the numerical results obtained by such an approach. Another way of 'switching' from level to level is refining until $h_{\min }$ becomes small enough (mesh becomes fine enough) to represent the current boundary layer $O(\varepsilon)$. The numerical testing of this approach is summarised in Table 9. We see that with the first approach, we are able to reach the error $[e]_{\overline{\mathrm{M}}}=1.49 e-02$ with 36625 d.o.f., whereas with the second approach we need 2951 d.o.f. (about 12 times less) to reach a similar error, namely $[e]_{\overline{\mathrm{M}}}=5.16 e-02$, on the homotopic level $\varepsilon=1 e-3$. Therefore, choosing the number of adaptive refinements based on the approximation or mesh properties seems to be the more advantageous approach. Such a strategy can be used as an efficient mesh-generation technique to provide an initial setting for strongly convection-dominated problems (see Figure 8). Here, each presented mesh corresponds to the final mesh on each of the $\varepsilon$ levels.

We also present convergence rates for the $L^{2}$-norm of the errors that has been addressed in [51. The obtained 


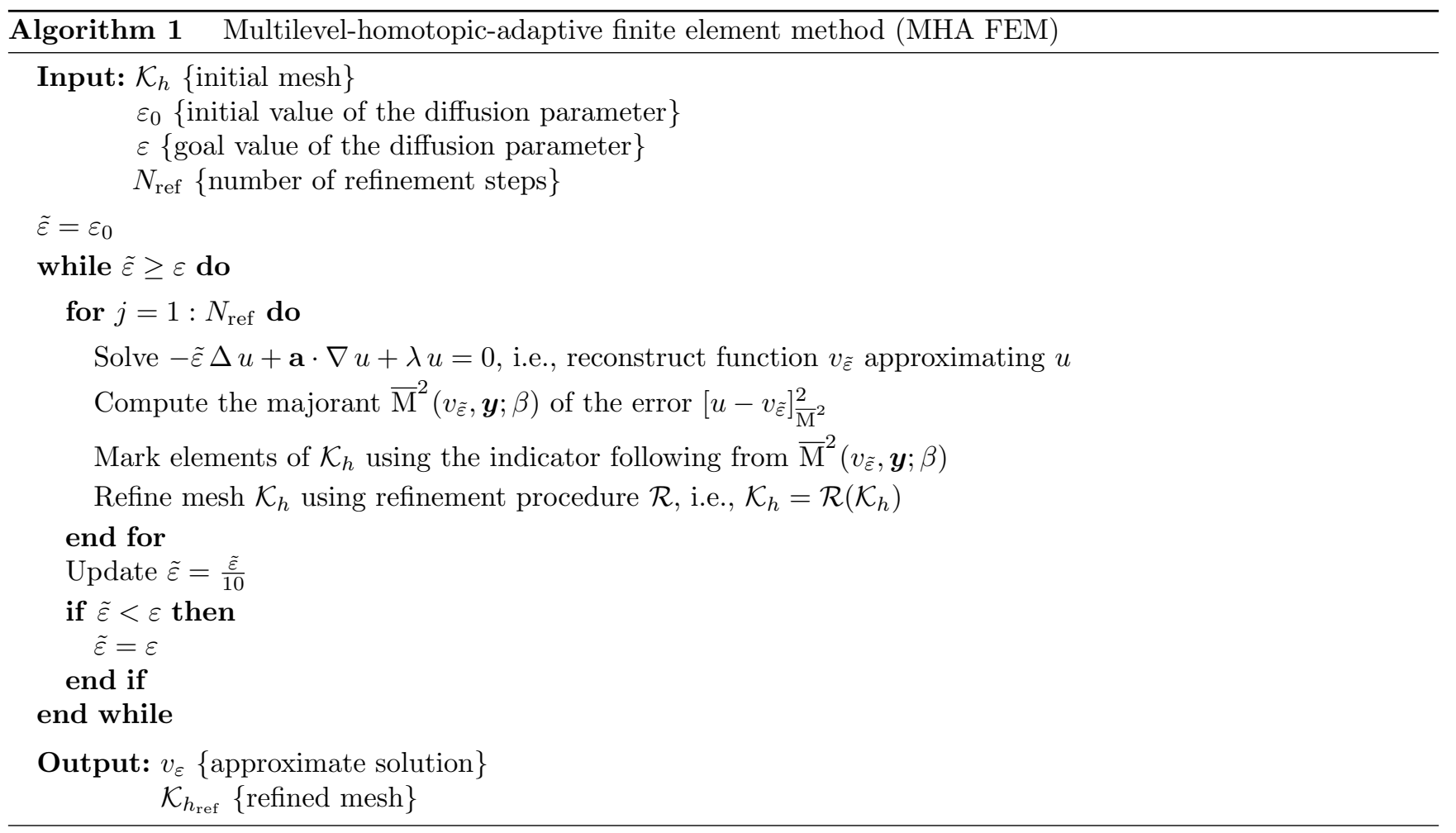

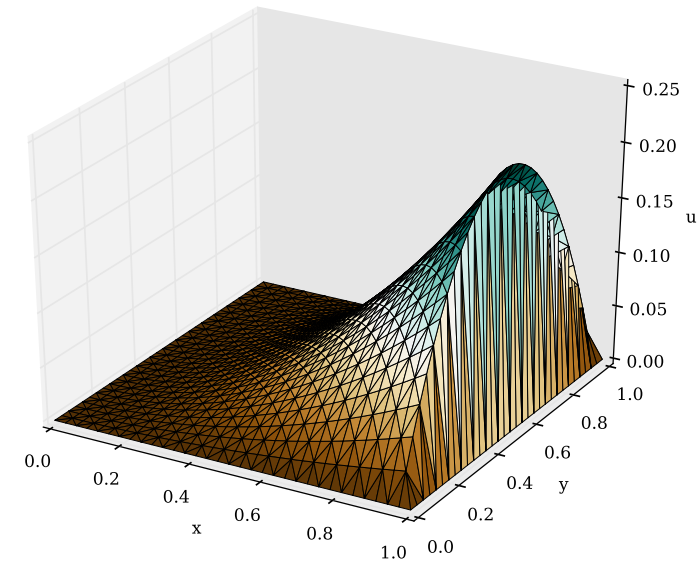

(a) $v \in P^{1}$, ref. 2: $1089 \mathrm{ND}, 2048 \mathrm{EL}$

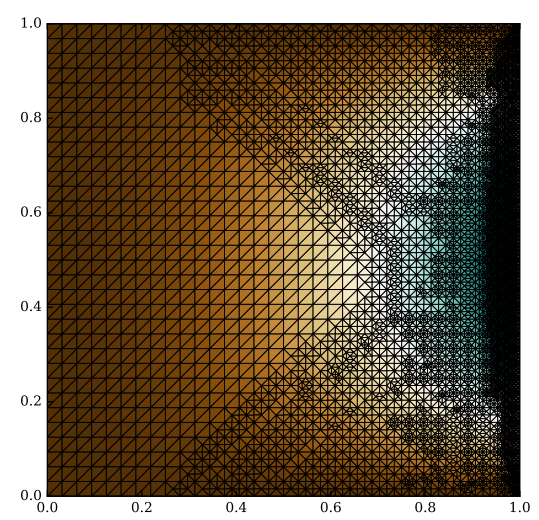

(b) $v \in P^{1}$, ref. 3: $13353 \mathrm{ND}, 26295 \mathrm{EL}$

Figure 7: Example 5. (a) Approximate solution on the mesh with 1089 nodes and 2048 elements obtained by uniform refinement. (b) Mesh with 6833 nodes and 13404 elements with approximate solution obtained by adaptive refinement.

numerical examples show that the $L^{2}$-error decreases as $O\left(h^{2}\right) \sim O\left(N^{-1}\right)$ (see Table 10 ).

Example 6 Let us also consider problem (67) (on which the MHA FEM algorithm was tested in [51, Example 4]). We now choose $d=2, \mathbf{a}=(2,3)^{\mathrm{T}}, \varepsilon=1 e-4, \lambda=1$, and the exact solution is defined by the function $u=\left(x y^{2}-y^{2} e^{2(x-1) / \varepsilon}-x e^{3(y-1) / \varepsilon}+e^{2(x-1)+3(y-1) / \varepsilon}\right)$ (it possesses regular boundary layers at $\{x=1\}$ and $\{y=1\}$ ).

First, we illustrate the behaviour of the majorant and minorant in Table 11. Table 12 provides the information on the $L_{2}$-error order of convergence. It is easy to observe that the obtained e.o.c. corresponds to the expected theoretical $O\left(h^{p+1}\right)$, which is $O\left(h^{2}\right)$ for linear Lagrangian elements. The level of the error $\|e\|_{L_{2}}:=1.97 e-05$ for 27817 d.o.f. is comparable to the $L^{2}$-error obtained in [51, i.e., $\|e\|_{L_{2}}:=8.38 e-05$ for 79104 d.o.f. $(\varepsilon=1 e-4)$. 


\begin{tabular}{|c|c|c|c|c|c|c|c|}
\hline ref. \# & d.o.f. \# & {$[e]_{\overline{\mathrm{M}}}$} & $\overline{\mathrm{M}}$ & $I_{\mathrm{eff}}(\overline{\mathrm{M}})$ & {$[e]_{\underline{\mathrm{M}}}$} & $\underline{\mathrm{M}}$ & $I_{\mathrm{eff}}(\underline{\mathrm{M}})$ \\
\hline \multicolumn{8}{|c|}{$\varepsilon=1$} \\
\hline 1 & 120 & $3.19 \mathrm{e}-02$ & $3.47 \mathrm{e}-02$ & 1.09 & $3.20 \mathrm{e}-02$ & $3.17 \mathrm{e}-02$ & 0.99 \\
\hline 2 & 183 & $2.48 \mathrm{e}-02$ & $2.76 \mathrm{e}-02$ & 1.11 & $2.49 \mathrm{e}-02$ & $2.46 \mathrm{e}-02$ & 0.99 \\
\hline 3 & 269 & $1.87 \mathrm{e}-02$ & $2.03 \mathrm{e}-02$ & 1.08 & $1.88 \mathrm{e}-02$ & $1.86 \mathrm{e}-02$ & 0.99 \\
\hline 4 & 403 & $1.62 \mathrm{e}-02$ & $1.80 \mathrm{e}-02$ & 1.11 & $1.63 \mathrm{e}-02$ & $1.62 \mathrm{e}-02$ & 0.99 \\
\hline \multicolumn{8}{|c|}{$\varepsilon=1 e-1$} \\
\hline 1 & 553 & $1.75 \mathrm{e}-02$ & $2.33 \mathrm{e}-02$ & 1.34 & $2.50 \mathrm{e}-02$ & $1.95 \mathrm{e}-02$ & 0.78 \\
\hline 2 & 804 & $1.24 \mathrm{e}-02$ & $1.65 \mathrm{e}-02$ & 1.33 & $1.88 \mathrm{e}-02$ & $1.41 \mathrm{e}-02$ & 0.75 \\
\hline 3 & 1214 & $1.01 \mathrm{e}-02$ & $1.34 \mathrm{e}-02$ & 1.32 & $1.52 \mathrm{e}-02$ & $1.14 \mathrm{e}-02$ & 0.75 \\
\hline 4 & 1841 & $8.26 \mathrm{e}-03$ & $1.08 \mathrm{e}-02$ & 1.31 & $1.24 \mathrm{e}-02$ & $9.26 \mathrm{e}-03$ & 0.75 \\
\hline \multicolumn{8}{|c|}{$\varepsilon=1 e-2$} \\
\hline 1 & 2556 & $2.67 \mathrm{e}-02$ & $5.89 \mathrm{e}-02$ & 2.21 & $4.01 \mathrm{e}-02$ & $3.79 \mathrm{e}-02$ & 0.94 \\
\hline 2 & 3652 & $1.75 \mathrm{e}-02$ & $4.14 \mathrm{e}-02$ & 2.36 & $2.80 \mathrm{e}-02$ & $2.61 \mathrm{e}-02$ & 0.93 \\
\hline 3 & 5494 & $1.33 \mathrm{e}-02$ & $3.17 \mathrm{e}-02$ & 2.39 & $2.14 \mathrm{e}-02$ & $1.98 \mathrm{e}-02$ & 0.93 \\
\hline 4 & 8132 & $1.06 \mathrm{e}-02$ & $2.47 \mathrm{e}-02$ & 2.32 & $1.67 \mathrm{e}-02$ & $1.55 \mathrm{e}-02$ & 0.93 \\
\hline \multicolumn{8}{|c|}{$\varepsilon=1 e-3$} \\
\hline 1 & 11311 & $3.60 \mathrm{e}-02$ & $8.07 \mathrm{e}-02$ & 2.24 & $5.69 \mathrm{e}-02$ & $5.65 \mathrm{e}-02$ & 0.99 \\
\hline 2 & 16294 & $2.40 \mathrm{e}-02$ & $6.24 \mathrm{e}-02$ & 2.59 & $4.07 \mathrm{e}-02$ & $4.03 \mathrm{e}-02$ & 0.99 \\
\hline 3 & 24662 & $1.98 \mathrm{e}-02$ & $4.81 \mathrm{e}-02$ & 2.43 & $3.15 \mathrm{e}-02$ & $3.11 \mathrm{e}-02$ & 0.99 \\
\hline 4 & 36625 & $1.49 \mathrm{e}-02$ & $3.96 \mathrm{e}-02$ & 2.65 & $2.46 \mathrm{e}-02$ & $2.42 \mathrm{e}-02$ & 0.98 \\
\hline
\end{tabular}

Table 8: Example 5. Majorant, minorant, and corresponding efficiency indices. Bulk marking with parameters $\theta=0.1,4$ refinements for each $\varepsilon$.

\begin{tabular}{|c|c|c|c|c|c|c|c|}
\hline ref. \# & d.o.f. \# & {$[e]_{\overline{\mathrm{M}}}$} & $\overline{\mathrm{M}}$ & $I_{\mathrm{eff}}(\overline{\mathrm{M}})$ & {$[e]_{\underline{\mathrm{M}}}$} & $\underline{\mathrm{M}}$ & $I_{\mathrm{eff}}(\underline{\mathrm{M}})$ \\
\hline \multicolumn{8}{|c|}{$\varepsilon=1$} \\
\hline 0 & 81 & $3.67 \mathrm{e}-02$ & $3.71 \mathrm{e}-02$ & 1.01 & $3.68 \mathrm{e}-02$ & $3.65 \mathrm{e}-02$ & 0.99 \\
\hline \multicolumn{8}{|c|}{$\varepsilon=1 e-1$} \\
\hline 0 & 120 & $5.00 \mathrm{e}-02$ & $5.95 \mathrm{e}-02$ & 1.19 & 7.32e-02 & $5.68 \mathrm{e}-02$ & 0.78 \\
\hline \multicolumn{8}{|c|}{$\varepsilon=1 e-2$} \\
\hline 0 & 170 & $1.01 \mathrm{e}-01$ & $2.12 \mathrm{e}-01$ & 2.11 & $2.34 \mathrm{e}-01$ & $2.07 \mathrm{e}-01$ & 0.88 \\
\hline 1 & 250 & $8.22 \mathrm{e}-02$ & $1.42 \mathrm{e}-01$ & 1.72 & $1.52 \mathrm{e}-01$ & $1.40 \mathrm{e}-01$ & 0.92 \\
\hline 2 & 362 & $5.97 \mathrm{e}-02$ & $9.43 \mathrm{e}-02$ & 1.58 & $1.00 \mathrm{e}-01$ & $9.26 \mathrm{e}-02$ & 0.92 \\
\hline 3 & 549 & $4.13 \mathrm{e}-02$ & $6.57 \mathrm{e}-02$ & 1.59 & $6.99 \mathrm{e}-02$ & $6.40 \mathrm{e}-02$ & 0.92 \\
\hline \multicolumn{8}{|c|}{$\varepsilon=1 e-3$} \\
\hline 0 & 816 & $9.96 \mathrm{e}-02$ & $2.31 \mathrm{e}-01$ & 2.32 & $2.29 \mathrm{e}-01$ & $2.25 \mathrm{e}-01$ & 0.98 \\
\hline 1 & 1196 & $8.22 \mathrm{e}-02$ & $1.67 \mathrm{e}-01$ & 2.03 & $1.65 \mathrm{e}-01$ & $1.61 \mathrm{e}-01$ & 0.98 \\
\hline 2 & 1914 & $6.52 \mathrm{e}-02$ & $1.25 \mathrm{e}-01$ & 1.91 & $1.23 \mathrm{e}-01$ & $1.19 \mathrm{e}-01$ & 0.97 \\
\hline 3 & 2951 & $5.16 \mathrm{e}-02$ & $9.71 \mathrm{e}-02$ & 1.88 & $9.50 \mathrm{e}-02$ & $9.16 \mathrm{e}-02$ & 0.96 \\
\hline \multicolumn{8}{|c|}{$\varepsilon=1 e-4$} \\
\hline 0 & 4450 & $1.05 \mathrm{e}-01$ & $2.97 \mathrm{e}-01$ & 2.84 & $2.90 \mathrm{e}-01$ & $2.79 \mathrm{e}-01$ & 0.96 \\
\hline 1 & 6825 & $9.33 \mathrm{e}-02$ & $2.31 \mathrm{e}-01$ & 2.47 & $2.28 \mathrm{e}-01$ & $2.13 \mathrm{e}-01$ & 0.94 \\
\hline 2 & 10540 & $8.19 \mathrm{e}-02$ & $1.77 \mathrm{e}-01$ & 2.16 & $1.76 \mathrm{e}-01$ & $1.64 \mathrm{e}-01$ & 0.93 \\
\hline 3 & 15817 & $6.70 \mathrm{e}-02$ & $1.36 \mathrm{e}-01$ & 2.04 & $1.34 \mathrm{e}-01$ & $1.25 \mathrm{e}-01$ & 0.94 \\
\hline 4 & 25318 & $5.41 \mathrm{e}-02$ & $1.04 \mathrm{e}-01$ & 1.92 & $1.02 \mathrm{e}-01$ & $9.76 \mathrm{e}-02$ & 0.95 \\
\hline 5 & 37928 & $4.30 \mathrm{e}-02$ & $8.18 \mathrm{e}-02$ & 1.90 & $8.18 \mathrm{e}-02$ & $7.75 \mathrm{e}-02$ & 0.95 \\
\hline \multicolumn{8}{|c|}{$\varepsilon=1 e-5$} \\
\hline 0 & 57437 & $1.01 \mathrm{e}-01$ & $2.69 \mathrm{e}-01$ & 2.66 & $2.64 \mathrm{e}-01$ & $2.58 \mathrm{e}-01$ & 0.97 \\
\hline 1 & 86256 & $9.11 \mathrm{e}-02$ & $2.06 \mathrm{e}-01$ & 2.26 & $2.05 \mathrm{e}-01$ & $1.95 \mathrm{e}-01$ & 0.95 \\
\hline 2 & 128442 & $7.47 \mathrm{e}-02$ & $1.54 \mathrm{e}-01$ & 2.06 & $1.56 \mathrm{e}-01$ & $1.45 \mathrm{e}-01$ & 0.93 \\
\hline 3 & 197588 & $6.10 \mathrm{e}-02$ & $1.17 \mathrm{e}-01$ & 1.93 & $1.17 \mathrm{e}-01$ & $1.12 \mathrm{e}-01$ & 0.95 \\
\hline
\end{tabular}

Table 9: Example 5. Majorant, minorant, and corresponding efficiency indices for different homotopic levels for bulk parameter $\theta=0.1$. 

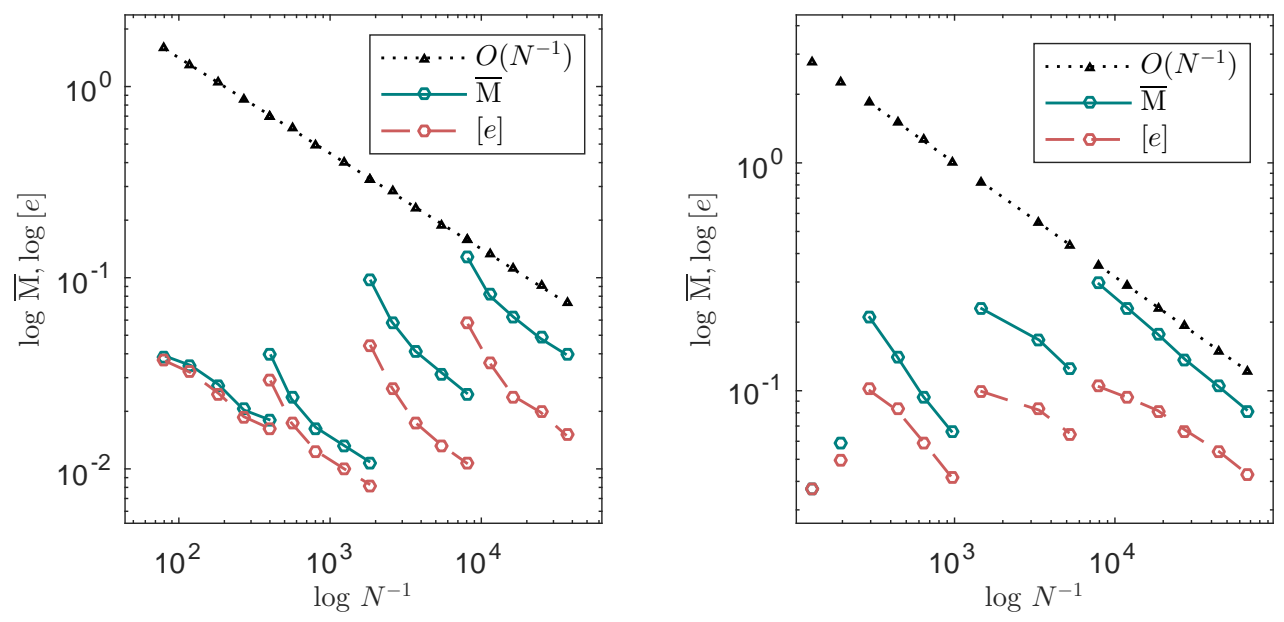

Figure 8: Example 5. Convergence of the error, majorant, and minorant corresponding to (a) Table 8 and (b) Table 9 .
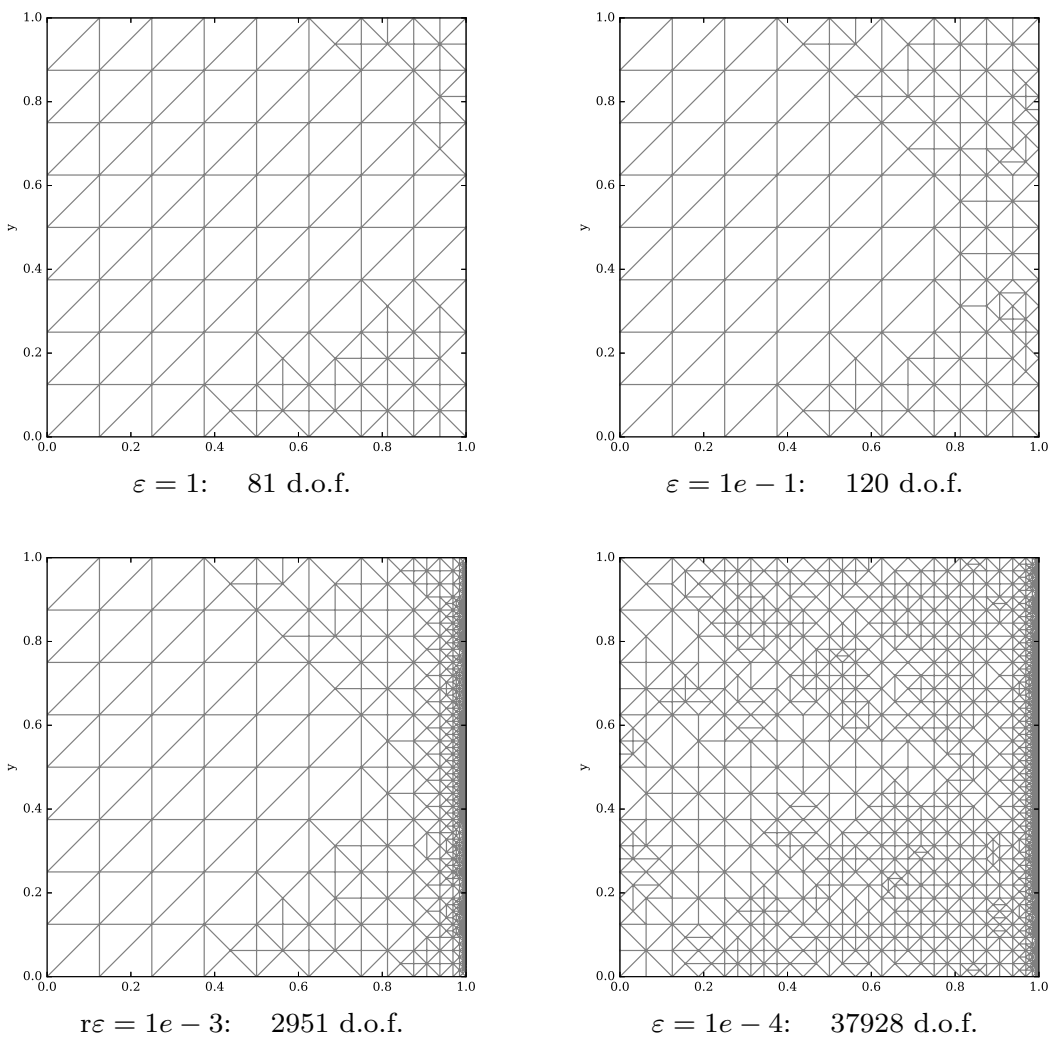

Figure 9: Example 5. Mesh obtained on the different homotopy levels using error indicators following from $\overline{\mathrm{M}}_{\mu_{\mathrm{opt}}}$ for $v \in \mathrm{P}_{1}, \boldsymbol{y} \in \mathrm{P}_{2}$ with bulk marking $\theta=0.1$.

However, in our experiment this accuracy is obtained for 2.8 times less d.o.f. then in [51]. We also present the mesh obtained by the adaptive refinement (driven by the indicator, which yields from the majorant), see Figure $10 \mathrm{~b}$ From the comparison of Figure 10b to Figure 9 in [51, Example 5], it is easy to see that in our case the refinement is rather concentrated on the region with a boundary layer rather than the region, where solution is smooth. 


\begin{tabular}{|c|c|c|c|c|}
\hline$N_{i}$ & {$[e]_{i}$} & $r\left([e]_{i}\right)=\frac{\ln \left([e]_{i} /[e]_{i+1}\right)}{\ln \left(N_{i}^{-1 / d} / N_{i+1}^{-1 / d}\right)}$ & $\|e\|_{L_{2}}$ & $r\left(\|e\|_{L_{2}}\right)$ \\
\hline \multicolumn{5}{|c|}{$\varepsilon=1 e-2$} \\
\hline 170 & $1.01 \mathrm{e}-01$ & 1.06 & $4.46 \mathrm{e}-04$ & 5.16 \\
\hline 250 & $8.22 \mathrm{e}-02$ & 1.72 & $1.65 \mathrm{e}-04$ & 5.04 \\
\hline 362 & $5.97 \mathrm{e}-02$ & 1.77 & $6.48 \mathrm{e}-05$ & 3.42 \\
\hline \multicolumn{5}{|c|}{$\varepsilon=1 e-3$} \\
\hline 816 & $9.96 \mathrm{e}-02$ & 1.00 & $4.27 \mathrm{e}-05$ & 3.82 \\
\hline 1196 & $8.22 \mathrm{e}-02$ & 0.99 & $2.06 \mathrm{e}-05$ & 2.76 \\
\hline 1914 & $6.52 \mathrm{e}-02$ & 1.08 & $1.08 \mathrm{e}-05$ & 2.44 \\
\hline \multicolumn{5}{|c|}{$\varepsilon=1 e-4$} \\
\hline 4450 & $1.05 \mathrm{e}-01$ & 0.53 & $7.30 \mathrm{e}-06$ & 2.46 \\
\hline 6825 & $9.33 \mathrm{e}-02$ & 0.60 & $4.31 \mathrm{e}-06$ & 2.61 \\
\hline 10540 & $8.19 \mathrm{e}-02$ & 0.99 & $2.44 \mathrm{e}-06$ & 2.97 \\
\hline 15817 & $6.70 \mathrm{e}-02$ & 0.91 & $1.34 \mathrm{e}-06$ & 2.45 \\
\hline 25318 & $5.41 \mathrm{e}-02$ & 1.13 & $7.53 \mathrm{e}-07$ & 2.19 \\
\hline
\end{tabular}

Table 10: Example 5. Convergence rates for the error $[e]$ and the $L_{2}$-error measure.
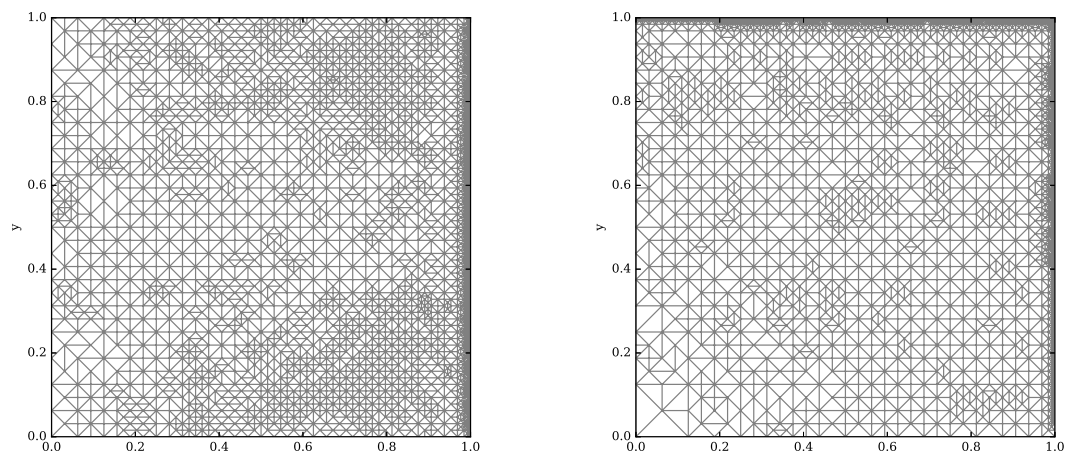

Figure 10: The final, adaptive mesh for (a) Example 5 and (b) Example 6 .

\subsection{Time-dependent problems}

In the next subsection, we consider the time-dependent reaction-convection-diffusion problem that follows from (19)- 222. We assume, that the balance equation (19) is written in the form

$$
u_{t}-\varepsilon \Delta u+\mathbf{a} \cdot \nabla u+\lambda u=0
$$

such that the reaction and convection are assumed to be independent of each other. Since the incremental timestepping method possesses several disadvantages, for instance, time-consuming sequentiality which leads to complications in the parallelisation, we use a variational space-time approach to solve $(19)-(22)$.

For our numerical examples, the generalised formulation (24) is discussed in the Petrov-Galerkin setting with different test and ansatz spaces (following the spirit of [49]): find

$$
u \in V_{u_{0}}:=\left\{v \in L_{2}\left(0, T ; H_{0}^{1}(\Omega)\right) \cap H^{1}\left(0, T ; H^{-1}(\Omega)\right) \mid v(x, 0)=u_{0}(x) \text { for } x \in \Omega\right\},
$$

such that

$$
\varepsilon(\nabla u, \nabla \eta)_{Q_{T}}+(\lambda u, \eta)_{Q_{T}}+(\mathbf{a} \cdot \nabla u, \eta)_{Q_{T}}+\left(u_{t}, \eta\right)_{Q_{T}}=: a(u, \eta)=\ell(\eta):=(f, \eta)_{Q_{T}},
$$

for all $\eta \in W:=L_{2}\left(0, T ; H_{0}^{1}(\Omega)\right)$. The stability condition is provided by the extension of [49, Theorem 2.1], i.e., having that $W \subset V_{u_{0}}$, the inequality

$$
c\|u\|_{V_{u_{0}}} \leq \sup _{v \in W, v \neq 0} \frac{a(u, v)}{\|v\|_{W}}
$$




\begin{tabular}{|c|c|c|c|c|c|c|c|}
\hline ref. \# & d.o.f. \# & {$[e]_{\overline{\mathrm{M}}}$} & $\overline{\mathrm{M}}$ & $I_{\mathrm{eff}}(\overline{\mathrm{M}})$ & {$[e]_{\underline{\mathrm{M}}}$} & $\underline{\mathrm{M}}$ & $I_{\text {eff }}(\underline{\mathrm{M}})$ \\
\hline \multicolumn{8}{|c|}{$\varepsilon=1$} \\
\hline 0 & 81 & $2.04 \mathrm{e}-02$ & $2.28 \mathrm{e}-02$ & 1.12 & $2.31 \mathrm{e}-02$ & $2.09 \mathrm{e}-02$ & 0.91 \\
\hline \multicolumn{8}{|c|}{$\varepsilon=1 e-1$} \\
\hline 0 & 119 & $4.45 \mathrm{e}-01$ & $6.65 \mathrm{e}-01$ & 1.49 & $8.98 \mathrm{e}-01$ & $6.09 \mathrm{e}-01$ & 0.68 \\
\hline \multicolumn{8}{|c|}{$\varepsilon=1 e-2$} \\
\hline 0 & 169 & $6.68 \mathrm{e}-01$ & $2.07 \mathrm{e}+00$ & 3.10 & $2.81 \mathrm{e}+00$ & $1.75 \mathrm{e}+00$ & 0.62 \\
\hline 1 & 241 & $6.53 \mathrm{e}-01$ & $1.54 \mathrm{e}+00$ & 2.36 & $2.08 \mathrm{e}+00$ & $1.36 \mathrm{e}+00$ & 0.65 \\
\hline 2 & 360 & $5.74 \mathrm{e}-01$ & $1.16 \mathrm{e}+00$ & 2.03 & $1.54 \mathrm{e}+00$ & $1.05 \mathrm{e}+00$ & 0.68 \\
\hline 3 & 559 & $4.96 \mathrm{e}-01$ & $8.91 \mathrm{e}-01$ & 1.80 & $1.14 \mathrm{e}+00$ & $8.20 \mathrm{e}-01$ & 0.72 \\
\hline \multicolumn{8}{|c|}{$\varepsilon=1 e-3$} \\
\hline 0 & 862 & $6.40 \mathrm{e}-01$ & $2.71 \mathrm{e}+00$ & 4.24 & $3.56 \mathrm{e}+00$ & $2.43 \mathrm{e}+00$ & 0.68 \\
\hline 1 & 1320 & $6.66 \mathrm{e}-01$ & $2.07 \mathrm{e}+00$ & 3.12 & $2.73 \mathrm{e}+00$ & $1.90 \mathrm{e}+00$ & 0.70 \\
\hline 2 & 2003 & $6.48 \mathrm{e}-01$ & $1.62 \mathrm{e}+00$ & 2.51 & $2.12 \mathrm{e}+00$ & $1.52 \mathrm{e}+00$ & 0.72 \\
\hline 3 & 3134 & $5.79 \mathrm{e}-01$ & $1.23 \mathrm{e}+00$ & 2.13 & $1.59 \mathrm{e}+00$ & $1.17 \mathrm{e}+00$ & 0.74 \\
\hline \multicolumn{8}{|c|}{$\varepsilon=1 e-4$} \\
\hline 0 & 4883 & $5.56 \mathrm{e}-01$ & $3.59 \mathrm{e}+00$ & 6.45 & $4.61 \mathrm{e}+00$ & $3.34 \mathrm{e}+00$ & 0.72 \\
\hline 1 & 7445 & $6.36 \mathrm{e}-01$ & $2.82 \mathrm{e}+00$ & 4.44 & $3.68 \mathrm{e}+00$ & $2.67 \mathrm{e}+00$ & 0.73 \\
\hline 2 & 11693 & $6.66 \mathrm{e}-01$ & $2.19 \mathrm{e}+00$ & 3.28 & $2.87 \mathrm{e}+00$ & $2.09 \mathrm{e}+00$ & 0.73 \\
\hline 3 & 17940 & $6.53 \mathrm{e}-01$ & $1.71 \mathrm{e}+00$ & 2.61 & $2.25 \mathrm{e}+00$ & $1.65 \mathrm{e}+00$ & 0.74 \\
\hline 4 & 27817 & $6.00 \mathrm{e}-01$ & $1.32 \mathrm{e}+00$ & 2.19 & $1.71 \mathrm{e}+00$ & $1.29 \mathrm{e}+00$ & 0.75 \\
\hline 5 & 43735 & $5.21 \mathrm{e}-01$ & $1.02 \mathrm{e}+00$ & 1.96 & $1.29 \mathrm{e}+00$ & $1.00 \mathrm{e}+00$ & 0.77 \\
\hline \multicolumn{8}{|c|}{$\varepsilon=1 e-5$} \\
\hline
\end{tabular}

Table 11: Example 6. Majorant, minorant, and corresponding efficiency indices for different homotopic levels with bulk parameter $\theta=0.1$.

\begin{tabular}{c|ccc}
$N_{i}$ & $\|e\|_{L_{2}}$ & $r\left(\|e\|_{L_{2}}\right):=\frac{\ln \left(\|e\|_{i} /\|e\|_{i+1}\right)}{\ln \left(N_{i}^{-1 / d} / N_{i+1}^{-1 / d}\right)}$ \\
\hline \multicolumn{3}{|c}{$\varepsilon=1 e-2$} \\
\hline 169 & $5.72 \mathrm{e}-03$ & 3.63 \\
241 & $3.01 \mathrm{e}-03$ & 3.26 \\
360 & $1.56 \mathrm{e}-03$ & 3.01 \\
\hline \multicolumn{3}{|c}{$\varepsilon=1 e-3$} \\
\hline 862 & $9.44 \mathrm{e}-04$ & 2.63 \\
1320 & $5.39 \mathrm{e}-04$ & 2.60 \\
2003 & $3.14 \mathrm{e}-04$ & 2.77 \\
\hline \multicolumn{3}{|c}{} \\
\hline 4883 & $1.61 \mathrm{e}-04$ & 2.20 \\
7445 & $1.01 \mathrm{e}-04$ & 2.31 \\
11693 & $6.00 \mathrm{e}-05$ & 2.45 \\
17940 & $3.55 \mathrm{e}-05$ & 2.68 \\
27817 & $1.97 \mathrm{e}-05$ & 2.67
\end{tabular}

Table 12: Example 6. Convergence rates of the $L_{2}$-norm.

holds with a positive constant $c$. After 'homogenisation' of the problem by splitting $u=\bar{u}(x, t)+\bar{u}_{0},(x, t) \in Q_{T}$, where $\bar{u}_{0}$ is an extension of $u_{0} \in H_{0}^{1}(\Omega)$ to the rest of the space-time cylinder. Hence, we aim to find $\bar{u} \in V_{0}$ such that

$$
a(\bar{u}, v)=<f, v_{h}>_{Q_{T}}-a\left(\bar{u}_{0}, v\right), \quad \forall v \in W .
$$

Using the fact that $V_{0} \subset W$, we can state existence and uniqueness of the solution of the weak formulation 70 based on [48, Theorem 3.7].

Let the discretisation spaces be $V_{h 0} \subset V_{0}$ and $W_{h} \subset W$ such that the inclusion $V_{h 0} \subset W_{h}$ is satisfied. Then, the 
discrete version of 70 can be presented as follows: find $\bar{u}_{h} \in V_{h 0}$ satisfying

$$
a\left(\bar{u}_{h}, v_{h}\right)=<f, v_{h}>_{Q_{T}}-a\left(\bar{u}_{0}, v_{h}\right), \quad \forall v_{h} \in W .
$$

The unique solvability of (71) follows from the discrete stability condition

$$
c\left\|u_{h}\right\|_{V_{h 0}} \leq \sup _{v_{h} \in W_{h}, v_{h} \neq 0} \frac{a\left(u_{h}, v_{h}\right)}{\left\|v_{h}\right\|_{W}}, \quad \forall u_{h} \in V_{h 0}, \quad c>0,
$$

which is proved in [49, Theorem 3.1].

Finally, we introduce the FE spaces for $u_{h} \in V_{h 0}$ and $w_{h} \in W_{h}$, i.e.,

$$
V_{h 0}=\mathrm{P}_{1}\left(Q_{h}\right) \cap V_{0}, \quad W_{h}=\mathrm{P}_{1}\left(Q_{h}\right) \cap W,
$$

where $\mathrm{P}_{1}\left(Q_{h}\right)$ is the FE space with a basis provided by piecewise linear and continuous functions (e.g., Lagrange polynomials). This brings us to the final approximation result for $\bar{u} \in V_{0}$ and $\bar{u}_{h} \in V_{h 0}$. According to 49, Theorem $3.3]$, by assuming that $u \in H^{2}(Q)$, we obtain the following energy error estimate

$$
\left\|\bar{u}-\bar{u}_{h}\right\|_{W} \leq \bar{c} h|u|_{H^{2}(Q)}
$$

with a $\bar{c}>0$ independent on $h$. More general estimate follows as a corollary [49, Corollary 3.4]. If $\bar{u} \in H_{0}^{s}\left(Q_{T}\right)$, $s \in[1,2]$, then the estimate

$$
\left\|\bar{u}-\bar{u}_{h}\right\|_{W} \leq \bar{C} h^{s-1}|\bar{u}|_{H_{0}^{s}\left(Q_{T}\right)}, \quad s \in[1, p+1],
$$

follows. Here, $\bar{C}$ is a generic positive constant, independent from the mesh parameter $h$. This theoretical result is confirmed by results of numerical tests (see Table 13 and 14).

Since the majorant is defined as an integral over the whole cylinder $Q_{T}$, it comes rather natural to use it for the error control. It also does not dependent on the discretisation used, therefore can be applied to the solutions reconstructed with fully unstructured grids. Next, we demonstrate numerical results obtained by applying functional type error estimates in case of a space-time discretisation.

Example 7 Consider a problem defined on the space-time cylinder $Q_{T}:=\Omega \times(0, T)$ such that $\Omega=(0,1), T=2$, $\partial \Omega=\Gamma_{N}$. For the diffusion-reaction-convection operator, see (68), we select $\varepsilon=1, \mathbf{a}=\mathbf{1}$, and three different cases for $\lambda(x, t)$ (see Figure 12 given as follows

$$
\begin{aligned}
& \text { (a) } \lambda(x, t)=\frac{1}{0.05 \sqrt{2 \pi}} e^{-200(x-0.2)^{2}}, \\
& \text { (b) } \lambda(x, t)=0.001(x+0.001)(t \sin t+1), \\
& \text { (c) } \lambda(x, t)=1000(x+0.001)(t \sin t+1) .
\end{aligned}
$$

The initial condition as well as the RHS are set to

$$
u_{0}=\sin 2 \pi+\cos \pi x \quad \text { and } \quad f=u_{0}(\cos (2 t)-2 t \sin (2 t))+\left(\pi^{2} \cos (\pi x)-\pi \sin \pi x+\lambda u_{0}\right)(t \cos 2 t+1) .
$$

The corresponding solution is illustrated in Figure 11 .

In order to provide robustness of the majorant w.r.t. changing values of $\lambda$, analogously to the elliptic case, we consider the additional function $\mu \in L_{[0,1]}^{\infty}\left(Q_{T}\right)$, which is used to balance the contribution of $\lambda$ into the $\mathbf{r}_{\mathrm{eq}}$ term, i.e.,

$$
\int_{Q_{T}} \mathbf{r}_{\text {eq }} e \mathrm{~d} x \mathrm{~d} t=\int_{Q_{T}} \mu \mathbf{r}_{\text {eq }} e \mathrm{~d} x \mathrm{~d} t+\int_{Q_{T}}(1-\mu) \mathbf{r}_{\text {eq }} e \mathrm{~d} x \mathrm{~d} t \leq\left\|\frac{\mu}{\delta} \mathbf{r}_{\text {eq }}\right\|_{\Omega}\|\delta e\|_{Q_{T}}+\frac{C_{\mathrm{F}}}{\sqrt{\varepsilon}}\left\|(1-\mu) \mathbf{r}_{\mathrm{eq}}\right\|_{Q_{T}}\|\nabla e\|_{\varepsilon, Q_{T}} .
$$

After setting parameters $\alpha_{1}=1+\beta, \alpha_{2}=1+\frac{1}{\beta}$, with $\beta=$ const, and $\alpha_{3}=2$ in $[58$, assuming that the initial conditions are satisfied exactly, i.e., $e(0, x)=0$, the final form of the estimate can be written as follows

$$
\begin{aligned}
\overline{\mathrm{M}}_{\mu}^{2}(v, \boldsymbol{y}) & :=\gamma\left\|\frac{\mu}{\sqrt{\lambda}} \mathbf{r}_{\mathrm{eq}}(v, \boldsymbol{y})\right\|_{Q_{T}}^{2}+\left(1+\frac{1}{\beta}\right) \frac{C_{\mathrm{F} \Omega}^{2}}{\varepsilon}\left\|(1-\mu) \mathbf{r}_{\mathrm{eq}}(v, \boldsymbol{y})\right\|_{Q_{T}}^{2}+(1+\beta)\left\|\mathbf{r}_{\mathrm{d}}(v, \boldsymbol{y})\right\|_{\varepsilon^{-1}, Q_{T}}^{2}+2 \frac{C_{\mathrm{tr}}^{2}}{\varepsilon}\|\boldsymbol{y} \cdot \mathbf{n}\|_{S_{T}}^{2} \\
& =\int_{Q_{T}}\left(\gamma \frac{\mu^{2}}{\lambda} \mathbf{r}_{\mathrm{eq}}^{2}(v, \boldsymbol{y})+\left(1+\frac{1}{\beta}\right) C_{\mathrm{F} \Omega}^{2}(1-\mu)^{2} \mathbf{r}_{\mathrm{eq}}^{2}(v, \boldsymbol{y})+(1+\beta) \varepsilon^{-1} \mathbf{r}_{\mathrm{d}}^{2}(v, \boldsymbol{y})\right) \mathrm{d} x \mathrm{~d} t+2 \frac{C_{\mathrm{tr}}^{2}}{\varepsilon}\|\boldsymbol{y} \cdot \mathbf{n}\|_{S_{T}}^{2} .
\end{aligned}
$$




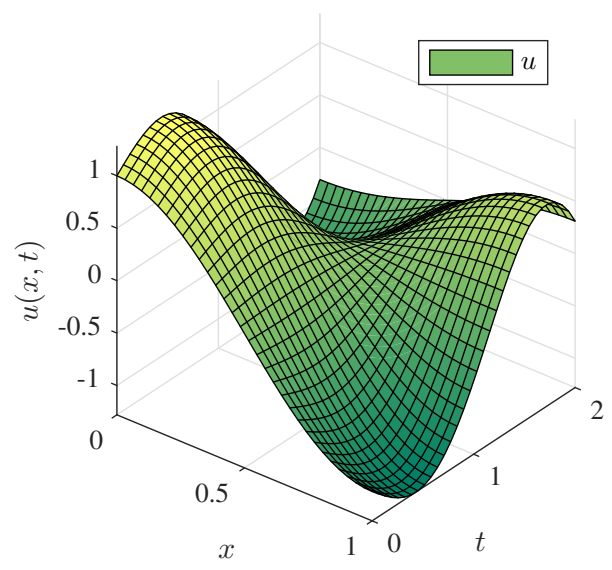

(a)

Figure 11: Example 7. The exact solution $u=(\sin 2 \pi+\cos (\pi x))(t \cos 2 t+1)$.

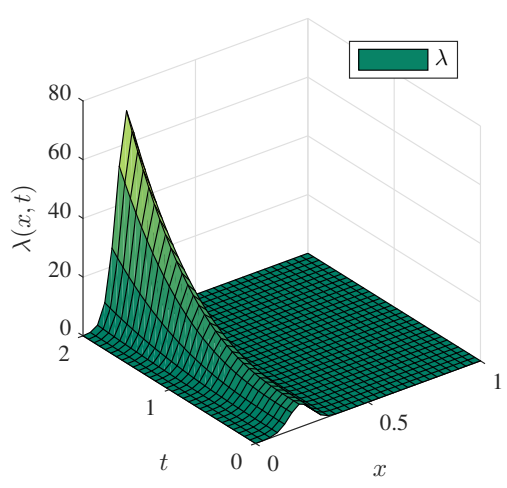

(a)

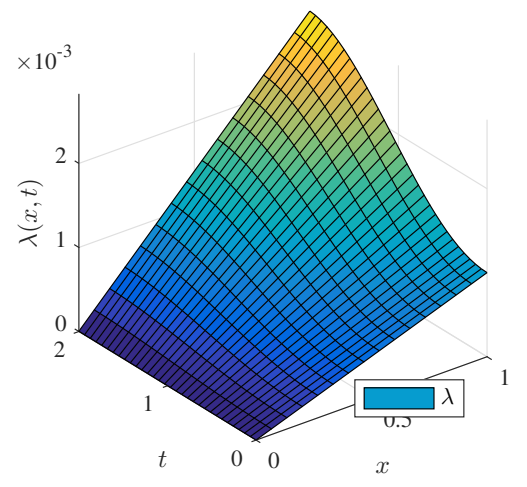

(b)

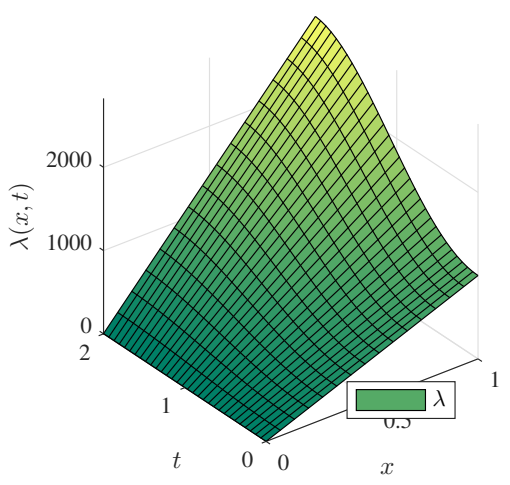

(c)

Figure 12: Example 7. Reaction functions $\lambda(x, t)$ for the case (a), (b), and (c).

\begin{tabular}{|c|c|c|c|c|c|c|}
\hline ref. & d.o.f. \# & {$[e]$} & $I_{\mathrm{eff}}\left(\overline{\mathrm{M}}_{\mu_{\mathrm{opt}}}\right)$ & d.o.f. \# & {$[e]$} & $I_{\mathrm{eff}}\left(\overline{\mathrm{M}}_{\mu=1}\right)$ \\
\hline & \multicolumn{6}{|c|}{ (a) $\lambda(x, t)=\frac{1}{0.05 \sqrt{2 \pi}} e^{-200(x-0.2)^{2}}$} \\
\hline 1 & 271 & $2.54 \mathrm{e}-01$ & 1.45 & 258 & $3.01 \mathrm{e}-01$ & $1.04 \cdot 1 \mathrm{e} 20$ \\
\hline 3 & 887 & $1.35 \mathrm{e}-01$ & 1.38 & 787 & $2.22 \mathrm{e}-01$ & $3.14 \cdot 1 \mathrm{e} 24$ \\
\hline 5 & 2864 & $7.40 \mathrm{e}-02$ & 1.37 & 2542 & $1.30 \mathrm{e}-01$ & $2.11 \cdot 1 \mathrm{e} 26$ \\
\hline 7 & 8692 & $4.10 \mathrm{e}-02$ & 1.39 & 8159 & $7.32 \mathrm{e}-02$ & $6.34 \cdot 1 \mathrm{e} 26$ \\
\hline \multirow[t]{2}{*}{9} & 25825 & $2.34 \mathrm{e}-02$ & 1.42 & 25425 & $3.90 \mathrm{e}-02$ & $1.03 \cdot 1 \mathrm{e} 26$ \\
\hline & \multicolumn{6}{|c|}{ (b) $\lambda(x, t)=0.001(x+0.001)(t \sin t+1)$} \\
\hline 1 & 272 & $2.53 \mathrm{e}-01$ & 1.49 & 257 & $3.00 \mathrm{e}-01$ & 39.65 \\
\hline 3 & 869 & $1.39 \mathrm{e}-01$ & 1.46 & 772 & $2.36 \mathrm{e}-01$ & 42.15 \\
\hline 5 & 2768 & $7.54 \mathrm{e}-02$ & 1.45 & 2411 & $1.64 \mathrm{e}-01$ & 56.30 \\
\hline 7 & 8450 & $4.15 \mathrm{e}-02$ & 1.48 & 7467 & $6.17 \mathrm{e}-02$ & 64.74 \\
\hline \multirow[t]{2}{*}{9} & 25240 & $2.38 \mathrm{e}-02$ & 1.57 & 22598 & $3.70 \mathrm{e}-02$ & 88.56 \\
\hline & \multicolumn{6}{|c|}{ (c) $\lambda(x, t)=1000(x+0.001)(t \sin t+1)$} \\
\hline 1 & 272 & $2.53 \mathrm{e}-01$ & 1.49 & 963 & $2.06 \mathrm{e}-02$ & 82.87 \\
\hline 3 & 869 & $1.39 \mathrm{e}-01$ & 1.46 & 2841 & $1.28 \mathrm{e}-02$ & 136.27 \\
\hline 5 & 2768 & $7.54 \mathrm{e}-02$ & 1.45 & 9111 & $8.59 \mathrm{e}-03$ & 213.33 \\
\hline 7 & 8450 & $4.15 \mathrm{e}-02$ & 1.48 & 27594 & $5.39 \mathrm{e}-03$ & 329.32 \\
\hline 9 & 25240 & $2.38 \mathrm{e}-02$ & 1.57 & 82646 & $1.18 \mathrm{e}-03$ & 1829.54 \\
\hline
\end{tabular}

Table 13: Example 7. Comparison of $\overline{\mathrm{M}}_{\mu_{\mathrm{opt}}}$ and $\overline{\mathrm{M}}_{\mu=1} v \in \mathrm{P}_{1}, \boldsymbol{y} \in \mathrm{P}_{2}$ with bulk marking $\theta=0.6$. 


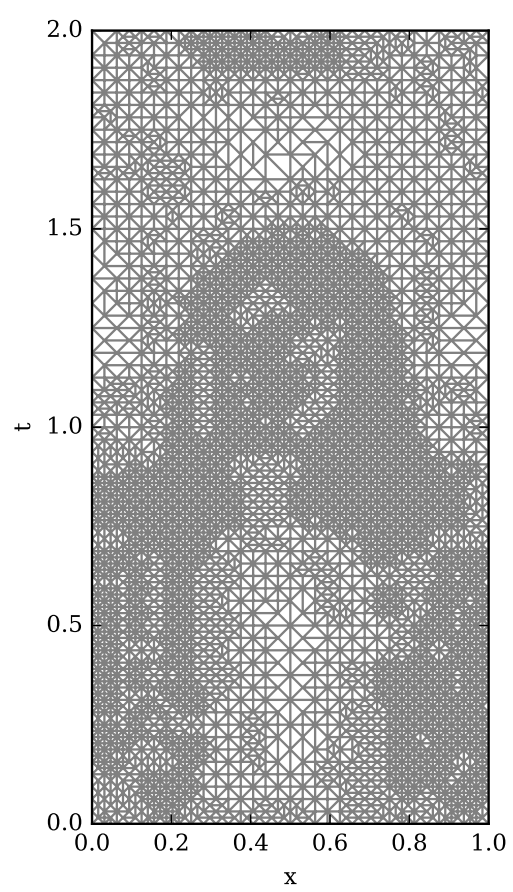

ref. based on $\overline{\mathrm{M}}_{\mu_{\mathrm{opt}}}$ for (a)

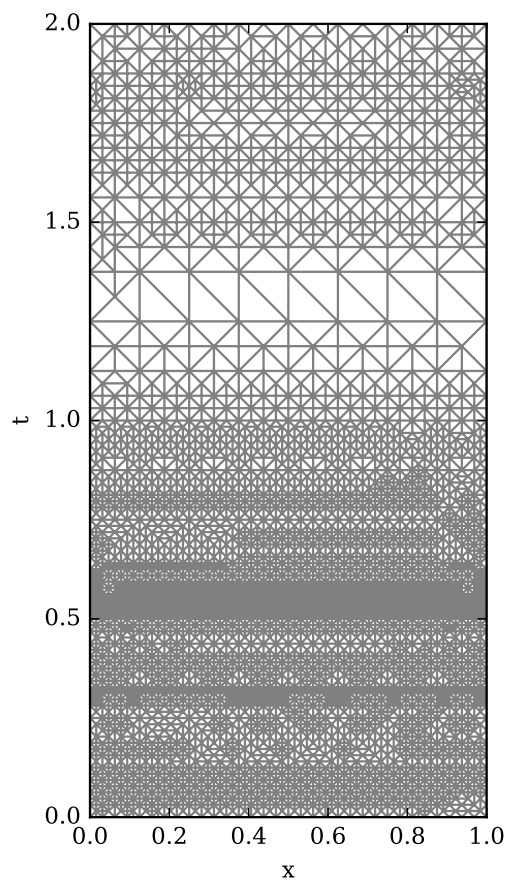

ref. based on $\overline{\mathrm{M}}_{\mu=1}$ for (a)

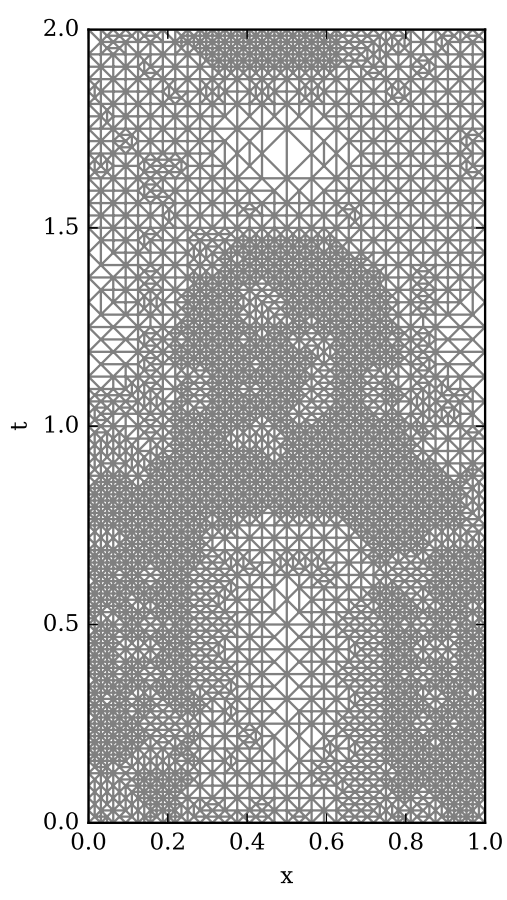

ref. based on $\overline{\mathrm{M}}_{\mu_{\mathrm{opt}}}$ for (b)

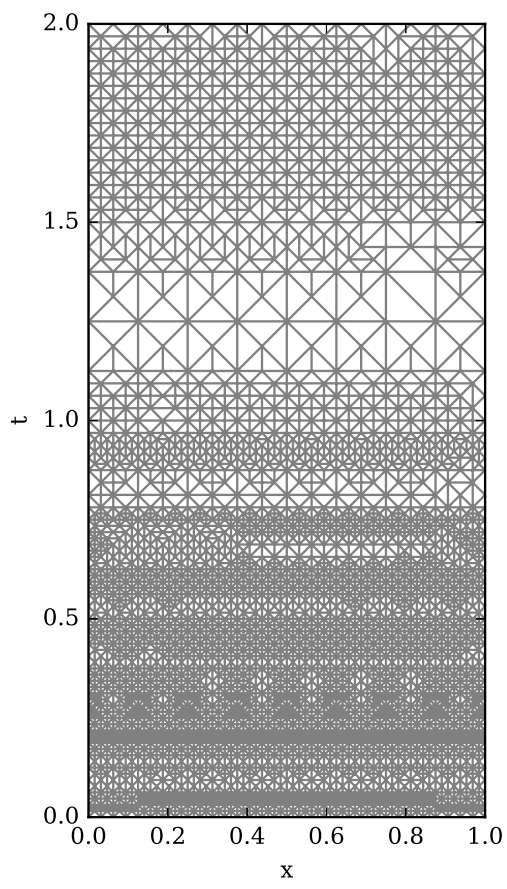

ref. based on $\overline{\mathrm{M}}_{\mu=1}$ for (b)

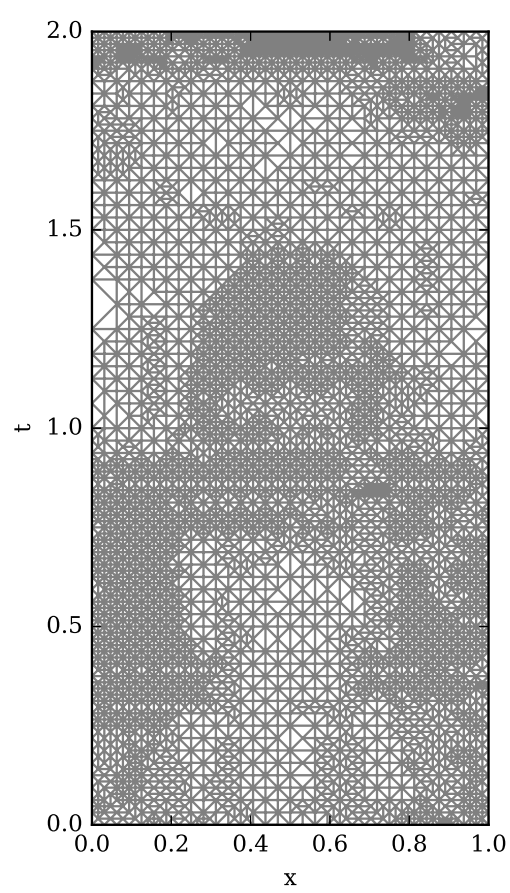

ref. based on $\overline{\mathrm{M}}_{\mu_{\mathrm{opt}}}$ for (c)

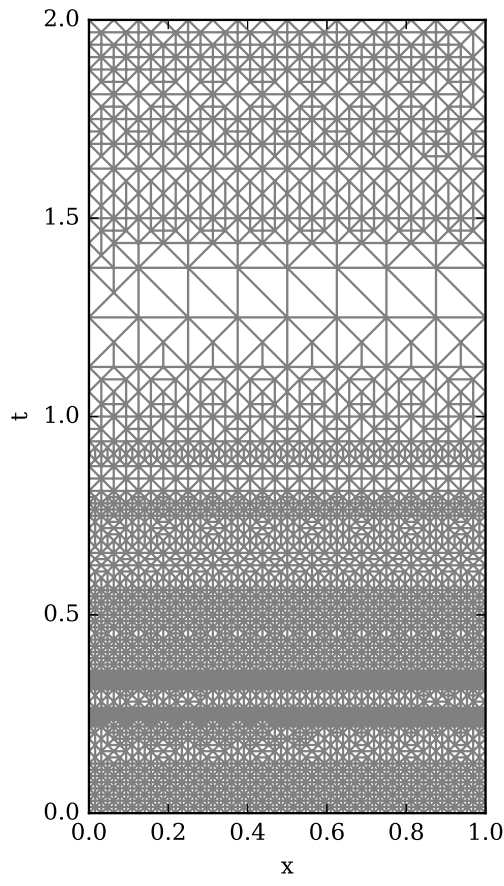

ref. based on $\overline{\mathrm{M}}_{\mu=1}$ for (c)

Figure 13: Example 7. The mesh obtained on the sixth refinement step using $\overline{\mathrm{M}}_{\mu_{\mathrm{opt}}}$ (first row) and $\overline{\mathrm{M}}_{\mu=1}$ (second row). Here, $v \in \mathrm{P}_{1}, \boldsymbol{y} \in \mathrm{P}_{2}$ with bulk marking $\theta=0.6$.

The minimisation of 77 w.r.t. $\mu$ is equivalent to the following variational problem: find $\hat{\mu} \in L_{[0,1]}^{\infty}\left(Q_{T}\right)$ such that

$$
\mu_{\mathrm{opt}}:=\underset{\mu \in L_{[0,1]}^{\infty}\left(Q_{T}\right)}{\operatorname{argmin}} \Upsilon(\mu), \quad \Upsilon(\mu):=\int_{Q_{T}}\left(\gamma \frac{\mu^{2}}{\lambda} \mathbf{r}_{\mathrm{eq}}^{2}(v, \boldsymbol{y})+\left(1+\frac{1}{\beta}\right) C_{\mathrm{F} \Omega}^{2}(1-\mu)^{2} \mathbf{r}_{\mathrm{eq}}^{2}(v, \boldsymbol{y})\right) \mathrm{d} x \mathrm{~d} t .
$$


Functional $\Upsilon(\mu)$ admits its minimum in $\mu_{\mathrm{opt}}(x, t)=\frac{C_{\mathrm{F} \Omega}^{2}(1+\beta) \lambda}{\beta \gamma+C_{\mathrm{F} \Omega}^{2}(1+\beta) \lambda}$. Table 13 provides the efficiency indices of $\overline{\mathrm{M}}_{\mu_{\mathrm{opt}}}$ and $\overline{\mathrm{M}}_{\mu=1}$ for different cases (a)-(b). The results correspond to the approximation $v \in \mathrm{P}_{1}$ and the auxiliary flux $\boldsymbol{y} \in \mathrm{P}_{2}$. The majorant $\overline{\mathrm{M}}_{\mu=1}$ grows dramatically in the case (a) and diverges according to the efficiency indices for the cases (b) and (c). At the same time, $\overline{\mathrm{M}}_{\mu_{\mathrm{opt}}}$ performs efficiently for all considered $\lambda$. It stays efficient and robust even if the reaction function changes its values drastically in different parts of the domain. The advantage of using an error estimate with optimal weighting function $\mu$ is illustrated also by the meshes in Figure 13 . Using $\overline{\mathrm{M}}_{\mu=1}$ in all cases (a)-(c) results in a refinement procedure generating meshes that do not make changes in the solution visible (see the lower row of Figure 13. The meshes presented in the upper row, however, reflect the approximate solution accurately.

If we assume that $\lambda=0$ and $\mathbf{a}=\mathbf{0}(\mathbf{F}=\mathbf{0}$ in the problem statement $(19)-(22))$, the test case reduces to the diffusion equation of parabolic type with right-hand side $f=u_{0}(\cos 2 t-2 t \sin 2 t)+\pi^{2} \cos \pi x(t \cos 2 t+1)$. Assuming that $f \in L_{2}(Q)$ and $u_{0} \in H_{0}^{1}\left(\Sigma_{0}\right)$ such a problem is uniquely solvable in $V_{0}^{\Delta_{x}}$, where

$$
V_{0}^{\Delta_{x}}:=\left\{v \in H^{1,1}\left(Q_{T}\right)\left|\Delta_{x} v \in L^{2}\left(Q_{T}\right) \wedge v\right|_{\Omega}=0 \text { for a.a. } t \in(0, T)\right\},
$$

and $u$ depends continuously on $t$ in the $H_{0}^{1}$ norm. If $u, v \in V_{0}^{\Delta_{x}}$ are provided, the time-dependent diffusion equation (19) imposes the error identity (see [1]):

$$
\left\|\operatorname{div}\left(\varepsilon \nabla_{x} e\right)\right\|_{Q_{T}}^{2}+\left\|\partial_{t} e\right\|_{Q_{T}}^{2}+\left\|\nabla_{x} e\right\|_{\varepsilon, \Sigma_{T}}^{2}=:\|e\|_{\mathcal{L}}^{2} \equiv \mathbb{E d}^{2}(v):=\left\|\nabla_{x}\left(u_{0}-v\right)\right\|_{\varepsilon, \Sigma_{0}}^{2}+\left\|\operatorname{div}\left(\varepsilon \nabla_{x} v\right)+f-\partial_{t} v\right\|_{Q_{T}}^{2} .
$$

The results corresponding to the performance of the majorant $\overline{\mathrm{M}}_{\mu_{\mathrm{opt}}}$ as well as $\mathbb{E d}$ in comparison to the errors they bound are illustrated in Table 14 . We see that for the setting (I) we get very close to the value one for the efficiency indices of $\overline{\mathrm{M}}_{\mu_{\mathrm{opt}}}$, and $\mathbb{E d}$ is always identical to the values of $\|e\|_{\mathcal{L}}^{2}$. However, an approximation of $v$ by linear affine elements does not provide the condition $v \in V_{0}^{\Delta_{x}}$ required for application of $\mathbb{E d}$. Therefore, stagnation of $\|e\|_{\mathcal{L}}^{2}$, as well as $\mathbb{E d}$, is expected. For the setting (II), we see that the strong norm of the error and the error identity begin to converge as $O(h)$. We also illustrate the results for the setting (III) in order to show that the performance of $\overline{\mathrm{M}}_{\mu_{\mathrm{opt}}}$ improves for smoother approximations of $\boldsymbol{y}$ (see the 5-th column). As we have observed in the numerical experiments, considering even smoother approximations of the flux does not pay off in this case, i.e., the minimisation of the majorant becomes even more time consuming, whereas the efficiency indices improve only slightly. The error identity stays quantitatively identical to the error norm $\|e\|_{\mathcal{L}}^{2}$ and, since it is only dependent on the approximation $v$, it does not require any computational time overhead. At the same time, the restriction on the regularity of $u, v \in V_{0}^{\Delta_{x}}$ still remains, which makes the application of the error identity rather restricted to a certain class of problems.

One of the ways to study the efficiency of the majorant when it comes to generating the mesh refinement procedure is to compare the numerical results for the refinement steps of adaptive meshes, where the refinement is based on the element-wise distribution of the majorant, with those meshes refined w.r.t. the the element-wise errors. Figure 14 provides such a comparison of meshes obtained on the refinement steps 4,6 , and 8 . We see that the meshes obtained in the upper row are topologically close to the meshes in the second row.

\section{Conclusions}

We have studied functional type a posteriori error estimation for stationary and time-dependent linear convectiondiffusion problems. This work was motivated by a decision-making Fokker-Planck model problem appearing in computational neuroscience, which has been discussed in [15]. We derived guaranteed two-sided estimates for the elliptic as well as parabolic problem and extended the a posteriori error analysis earlier derived in [41, 46] by studying the numerical aspects as well as properties of the two-sided error bounds applied to this class of problems.

We presented a set of various numerical examples for different parameters and boundary conditions showing the sharpness of the upper and lower bounds in practice. In particular, we addressed convection-dominated problems including adaptive mesh refinement based on a bulk marking criterion from [18. Furthermore, we presented numerical experiments applying the multilevel-homotopic-adaptive finite element method in the stationary case and space-time discretisation for the time-dependent reaction-convection-diffusion problem. The numerical results in both cases, static as well as time-dependent, successfully demonstrated the capability of the two-sided error bounds to provide a basis for the implementation of reliable and efficient computational methods. 


\begin{tabular}{|c|c|c|c|c|c|c|c|}
\hline ref. & d.o.f. \# & {$[e]$} & $\overline{\mathrm{M}}_{\mu_{\mathrm{opt}}}$ & $I_{\mathrm{eff}}\left(\overline{\mathrm{M}}_{\mu_{\mathrm{opt}}}\right)$ & $\|e\|$ & Ed & $I_{\text {eff }}(\mathbb{E d})$ \\
\hline \multicolumn{8}{|c|}{ (I) $v \in \mathrm{P}_{1}$ and $\boldsymbol{y} \in \mathrm{P}_{2}$} \\
\hline 1 & 272 & $2.54 \mathrm{e}-01$ & $3.84 \mathrm{e}-01$ & 1.51 & $8.42 \mathrm{e}+00$ & $8.42 \mathrm{e}+00$ & 1.00 \\
\hline 3 & 887 & $1.36 \mathrm{e}-01$ & $1.99 \mathrm{e}-01$ & 1.46 & $8.42 \mathrm{e}+00$ & $8.42 \mathrm{e}+00$ & 1.00 \\
\hline 5 & 2867 & $7.42 \mathrm{e}-02$ & $1.08 \mathrm{e}-01$ & 1.45 & $8.42 \mathrm{e}+00$ & $8.42 \mathrm{e}+00$ & 1.00 \\
\hline 7 & 8738 & $4.08 \mathrm{e}-02$ & $5.99 \mathrm{e}-02$ & 1.47 & $8.42 \mathrm{e}+00$ & $8.42 \mathrm{e}+00$ & 1.00 \\
\hline 9 & 26132 & $2.33 \mathrm{e}-02$ & $3.53 \mathrm{e}-02$ & 1.52 & $8.42 \mathrm{e}+00$ & $8.42 \mathrm{e}+00$ & 1.00 \\
\hline \multicolumn{8}{|c|}{ (II) $v \in \mathrm{P}_{2}$ and $\boldsymbol{y} \in \mathrm{P}_{2}$} \\
\hline 1 & 1033 & $1.44 \mathrm{e}-02$ & $7.60 \mathrm{e}-02$ & 5.26 & $8.08 \mathrm{e}-01$ & $8.08 \mathrm{e}-01$ & 1.00 \\
\hline 3 & 3672 & $4.09 \mathrm{e}-03$ & $1.83 \mathrm{e}-02$ & 4.49 & $4.28 \mathrm{e}-01$ & $4.28 \mathrm{e}-01$ & 1.00 \\
\hline 5 & 12382 & $1.14 \mathrm{e}-03$ & $5.35 \mathrm{e}-03$ & 4.70 & $2.32 \mathrm{e}-01$ & $2.32 \mathrm{e}-01$ & 1.00 \\
\hline 7 & 40392 & $3.28 \mathrm{e}-04$ & $1.53 \mathrm{e}-03$ & 4.67 & $1.27 \mathrm{e}-01$ & $1.27 \mathrm{e}-01$ & 1.00 \\
\hline 9 & 132711 & $9.92 \mathrm{e}-05$ & $4.74 \mathrm{e}-04$ & 4.78 & $6.85 \mathrm{e}-02$ & $6.85 \mathrm{e}-02$ & 1.00 \\
\hline \multicolumn{8}{|c|}{ (III) $v \in \mathrm{P}_{2}$ and $\boldsymbol{y} \in \mathrm{P}_{3}$} \\
\hline 1 & 1029 & $1.53 \mathrm{e}-02$ & $4.73 \mathrm{e}-02$ & 3.10 & $8.46 \mathrm{e}-01$ & $8.45 \mathrm{e}-01$ & 1.00 \\
\hline 3 & 3789 & $3.98 \mathrm{e}-03$ & $1.09 \mathrm{e}-02$ & 2.73 & $4.25 \mathrm{e}-01$ & $4.24 \mathrm{e}-01$ & 1.00 \\
\hline 5 & 12703 & $1.10 \mathrm{e}-03$ & $3.33 \mathrm{e}-03$ & 3.01 & $2.26 \mathrm{e}-01$ & $2.26 \mathrm{e}-01$ & 1.00 \\
\hline 7 & 41749 & $3.17 \mathrm{e}-04$ & $1.01 \mathrm{e}-03$ & 3.20 & $1.23 \mathrm{e}-01$ & $1.23 \mathrm{e}-01$ & 1.00 \\
\hline 9 & 141873 & $9.40 \mathrm{e}-05$ & $2.92 \mathrm{e}-04$ & 3.10 & $6.63 \mathrm{e}-02$ & $6.63 \mathrm{e}-02$ & 1.00 \\
\hline
\end{tabular}

Table 14: Example 7. Efficiency of $\overline{\mathrm{M}}_{\mu_{\mathrm{opt}}}$ and $\mathbb{E d}$ with bulk marking $\theta=0.6$.

\section{Acknowledgments}

The authors gratefully acknowledge the financial support by the Austrian Science Fund (FWF) through the NFN S117-03 project, and by the Academy of Finland, grant 295897.

\section{References}

[1] I. Anjam and D. Pauly. An elementary method of deriving a posteriori error equalities and estimates for linear partial differential equations. Computers and Mathematics with Applications (CMAM), 2017. accepted for publication, also available as arxiv-report with a number arxiv:math.NA:1612.01411.

[2] R. Araya, E. Behrens, and R. Rodrí guez. An adaptive stabilized finite element scheme for the advectionreaction-diffusion equation. Appl. Numer. Math., 54(3-4):491-503, 2005.

[3] W. Bangerth and R. Rannacher. Adaptive finite element methods for differential equations. Lectures in Mathematics ETH Zürich. Birkhäuser Verlag, Basel, 2003.

[4] R. E. Bank, J. F. Bürgler, W. Fichtner, and R. K. Smith. Some upwinding techniques for finite element approximations of convection-diffusion equations. Numer. Math., 58(2):185-202, 1990.

[5] E. Bänsch, P. Morin, and R. H. Nochetto. An adaptive Uzawa FEM for the Stokes problem: convergence without the inf-sup condition. SIAM J. Numer. Anal., 40(4):1207-1229, 2002.

[6] C. E. Baumann and J. T. Oden. A discontinuous $h p$ finite element method for the Euler and Navier-Stokes equations. Internat. J. Numer. Methods Fluids, 31(1):79-95, 1999. Tenth International Conference on Finite Elements in Fluids (Tucson, AZ, 1998).

[7] F. Brezzi, L. P. Franca, T. J. R. Hughes, and A. Russo. b= $b$ g. Comput. Methods Appl. Mech. Engrg., $145(3-4): 329-339,1997$.

[8] F. Brezzi, L. P. Franca, and A. Russo. Further considerations on residual-free bubbles for advective-diffusive equations. Comput. Methods Appl. Mech. Engrg., 166(1-2):25-33, 1998.

[9] F. Brezzi, T. J. R. Hughes, L. D. Marini, A. Russo, and E. Süli. A priori error analysis of residual-free bubbles for advection-diffusion problems. SIAM J. Numer. Anal., 36(6):1933-1948, 1999. 


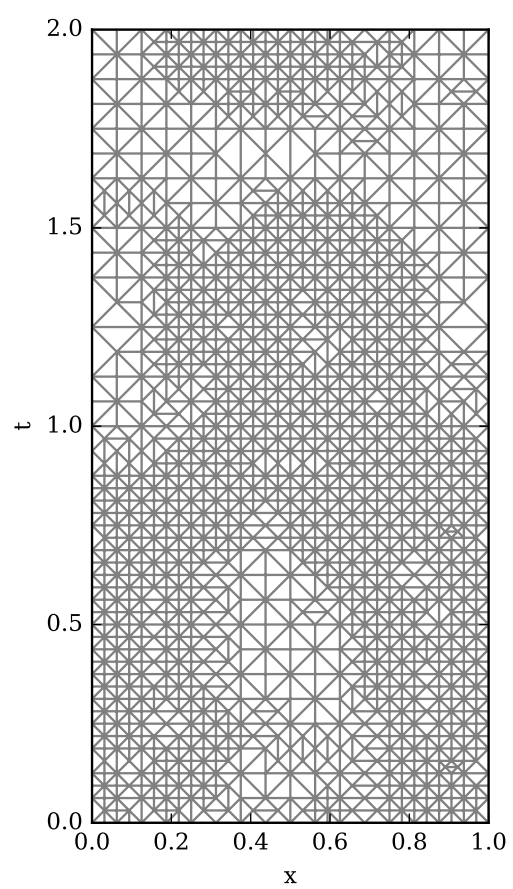

ref. 4: based on $\overline{\mathrm{M}}_{\mu_{\mathrm{opt}}}$

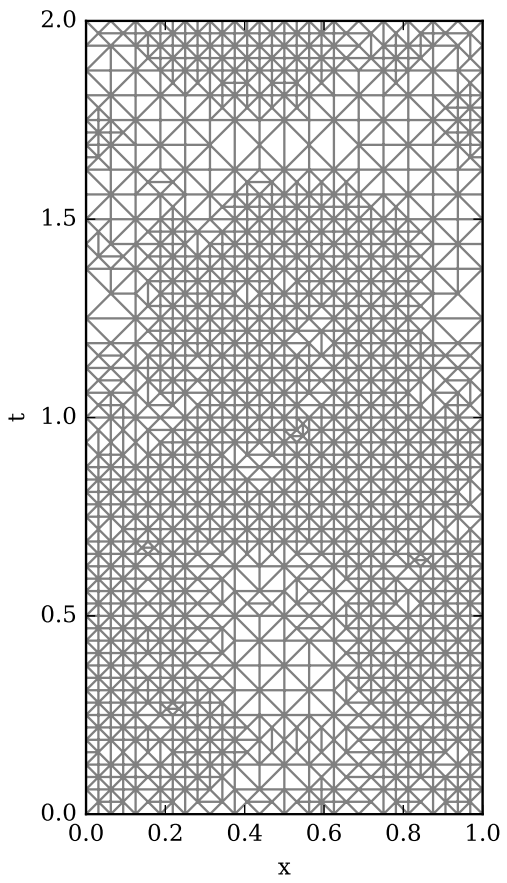

ref. 4: based on [e]

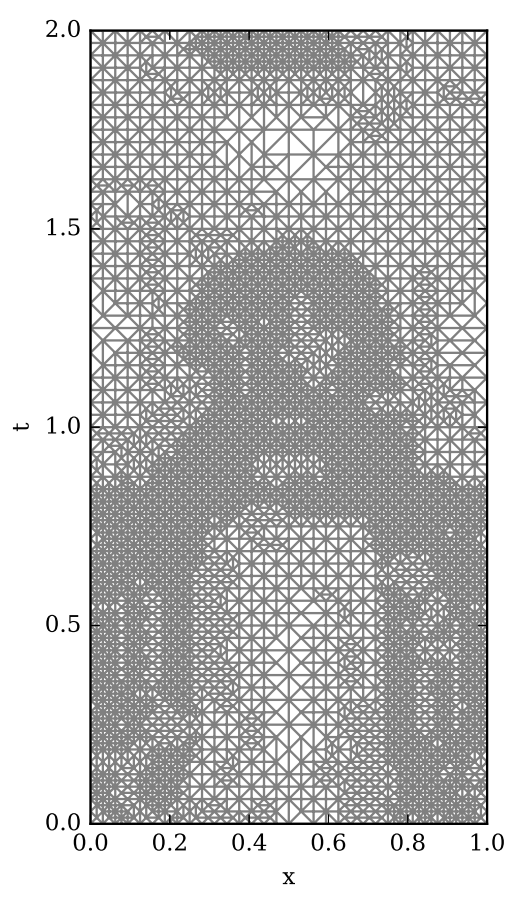

ref. 6: based on $\overline{\mathrm{M}}_{\mu_{\mathrm{opt}}}$

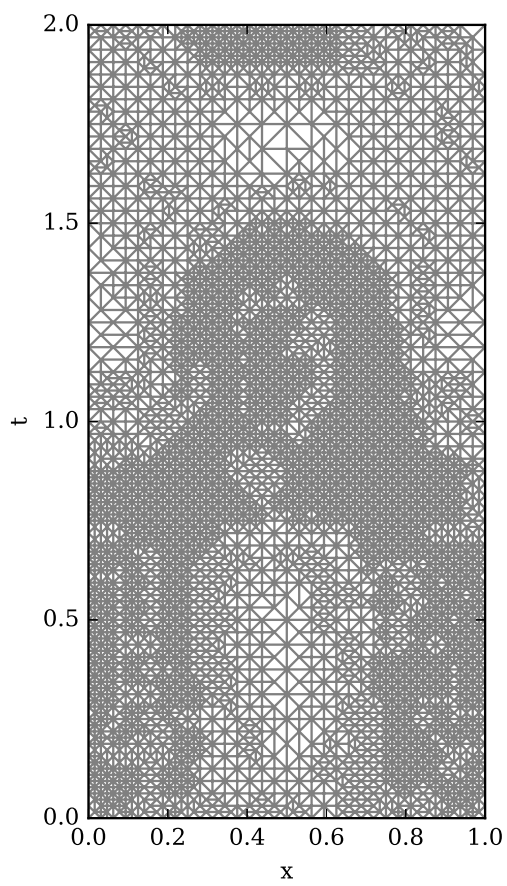

ref. 6: based on $[e]$

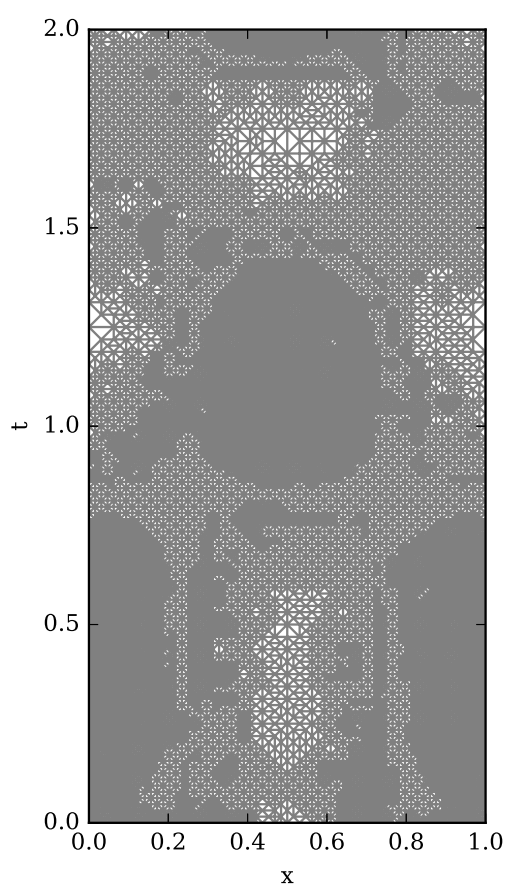

ref. 8: based on $\overline{\mathrm{M}}_{\mu_{\mathrm{opt}}}$

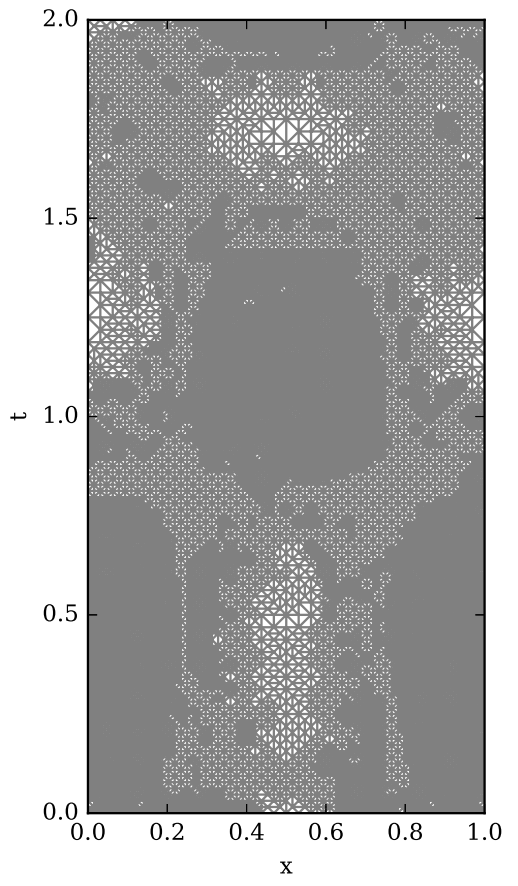

ref. 8: based on $[e]$

Figure 14: Example 7. The mesh obtained on the refinement steps (ref.) 4, 6, and 8 using $\overline{\mathrm{M}}_{\mu_{\mathrm{opt}}}$ (first row) and [e] (second row). Here, $v \in \mathrm{P}_{1}, \boldsymbol{y} \in \mathrm{P}_{2}$ with bulk marking $\theta=0.6$.

[10] F. Brezzi, D. Marini, and E. Süli. Residual-free bubbles for advection-diffusion problems: the general error analysis. Numer. Math., 85(1):31-47, 2000.

[11] F. Brezzi, L. D. Marini, and P. Pietra. Two-dimensional exponential fitting and applications to drift-diffusion 
models. SIAM J. Numer. Anal., 26(6):1342-1355, 1989.

[12] A. N. Brooks and T. J. R. Hughes. Streamline upwind/Petrov-Galerkin formulations for convection dominated flows with particular emphasis on the incompressible Navier-Stokes equations. Comput. Methods Appl. Mech. Engrg., 32(1-3):199-259, 1982. FENOMECH '81, Part I (Stuttgart, 1981).

[13] E. Brown and P. Holmes. Modeling a simple choice task: stochastic dynamics of mutually inhibitory neural groups. Stochastics and dynamics, 1(02):159-191, 2001.

[14] E. Burman and P. Hansbo. Edge stabilization for Galerkin approximations of convection-diffusion-reaction problems. Comput. Methods Appl. Mech. Engrg., 193(15-16):1437-1453, 2004.

[15] J. A. Carrillo, S. Cordier, and S. Mancini. A decision-making fokker-planck model in computational neuroscience. Journal of mathematical biology, 63(5):801-830, 2011.

[16] Long Chen, Pengtao Sun, and Jinchao Xu. Multilevel homotopic adaptive finite element methods for convection dominated problems. In Domain decomposition methods in science and engineering, volume 40 of Lect. Notes Comput. Sci. Eng., pages 459-468. Springer, Berlin, 2005.

[17] G. Deco and D. Martí. Deterministic analysis of stochastic bifurcations in multi-stable neurodynamical systems. Biological cybernetics, 96(5):487-496, 2007.

[18] W. Dörfler. A convergent adaptive algorithm for Poisson's equation. SIAM Journal on Numerical Analysis, 33(3):1106-1124, 1996.

[19] Jim Douglas, Jr. and Todd Dupont. Interior penalty procedures for elliptic and parabolic Galerkin methods. pages 207-216. Lecture Notes in Phys., Vol. 58, 1976.

[20] L. C. Evans. Partial differential equations, volume 19 of Graduate Studies in Mathematics. American Mathematical Society, Providence, RI, second edition, 2010.

[21] K. Friedrichs. On certain inequalities and characteristic value problems for analytic functions and for functions of two variables. Trans. Amer. Math. Soc., 41(3):321-364, 1937.

[22] J.C. Heinrich, P.S. Huyakorn, O.C. Zienkiewicz, and A.R. Mitchell. An 'upwind' finite element scheme for two-dimensional convective transport equation. Int. J. Numer. Methods Eng., 11:131-143, 1977.

[23] M. H. Holmes. Introduction to perturbation methods, volume 20 of Texts in Applied Mathematics. Springer, New York, second edition, 2013.

[24] P. Houston, C. Schwab, and E. Süli. Discontinuous $h p$-finite element methods for advection-diffusion-reaction problems. SIAM J. Numer. Anal., 39(6):2133-2163, 2002.

[25] T. J. R. Hughes, L. P. Franca, and G. M. Hulbert. new finite element formulation for computational fluid dynamics. viii. the galerkin/least-squares method for advective-diffusive equations. Comput. Methods Appl. Mech. Engrg., 73:173-189, 1989.

[26] V. John. A numerical study of a posteriori error estimators for convection-diffusion equations. Comput. Methods Appl. Mech. Engrg., 190(5-7):757-781, 2000.

[27] V. John and P. Knobloch. On spurious oscillations at layers diminishing (SOLD) methods for convectiondiffusion equations. I. A review. Comput. Methods Appl. Mech. Engrg., 196(17-20):2197-2215, 2007.

[28] V. John and P. Knobloch. On spurious oscillations at layers diminishing (SOLD) methods for convectiondiffusion equations. II. Analysis for $P_{1}$ and $Q_{1}$ finite elements. Comput. Methods Appl. Mech. Engrg., 197(2124):1997-2014, 2008.

[29] T. Kang and D. Yu. Some a posteriori error estimates of the finite-difference streamline-diffusion method for convection-dominated diffusion equations. Adv. Comput. Math., 15(1-4):193-218 (2002), 2001. A posteriori error estimation and adaptive computational methods.

[30] R. Lazarov and S. Tomov. A posteriori error estimates for finite volume element approximations of convectiondiffusion-reaction equations. Computational Geosciences, 6(3-4):483-503, 2002. 
[31] R. Li, T. Tang, and P. Zhang. A moving mesh finite element algorithm for singular problems in two and three space dimensions. J. Comput. Phys., 177(2):365-393, 2002.

[32] A. Logg, K.-A. Mardal, and G. N. Wells, editors. Automated solution of differential equations by the finite element method, volume 84 of Lecture Notes in Computational Science and Engineering. Springer, Heidelberg, 2012. The FEniCS book.

[33] O. Mali, P. Neittaanmäki, and S. Repin. Accuracy verification methods, volume 32 of Computational Methods in Applied Sciences. Springer, Dordrecht, 2014. Theory and algorithms.

[34] S. Matculevich and S. Repin. Computable estimates of the distance to the exact solution of the evolutionary reaction-diffusion equation. Appl. Math. Comput., 247:329-347, 2014.

[35] P. Neittaanmäki and S. Repin. Reliable methods for computer simulation, volume 33 of Studies in Mathematics and its Applications. Elsevier Science B.V., Amsterdam, 2004. Error control and a posteriori estimates.

[36] H. Poincare. Sur les Equations aux Derivees Partielles de la Physique Mathematique. Amer. J. Math., 12(3):211-294, 1890.

[37] H. Poincare. Sur les Equations de la Physique Mathematique. Rend. Circ. Mat. Palermo, 8:57-156, 1894.

[38] S. Repin. A posteriori error estimation for variational problems with power growth functionals based on duality theory. Zapiski Nauchnych Seminarov POMI, 249:244-255, 1997.

[39] S. Repin. A posteriori estimates for approximate solutions of variational problems with strongly convex functionals. Problems of Mathematical Analysis, 17:199-226, 1997.

[40] S. Repin. A posteriori error estimation for variational problems with uniformly convex functionals. Math. Comput., 69(230):481-500, 2000.

[41] S. Repin. A posteriori estimates for partial differential equations, volume 4 of Radon Series on Computational and Applied Mathematics. Walter de Gruyter GmbH \& Co. KG, Berlin, 2008.

[42] S. Repin, S. Sauter, and A. Smolianski. Two-sided a posteriori error estimates for mixed formulations of elliptic problems. SIAM J. Numer. Anal., 45(3):928-945, 2007.

[43] S. Repin and A. Smolianski. Functional-type a posteriori error estimates for mixed finite element methods. Russian J. Numer. Anal. Math. Modelling, 20(4):365-382, 2005.

[44] S. I. Repin. A unified approach to a posteriori error estimation based on duality error majorants. Math. Comput. Simulation, 50(1-4):305-321, 1999. Modelling '98 (Prague).

[45] S. I. Repin. Estimates of deviations from exact solutions of initial-boundary value problem for the heat equation. Rend. Mat. Acc. Lincei, 13(9):121-133, 2002.

[46] S. I. Repin and S. K. Tomar. A posteriori error estimates for approximations of evolutionary convectiondiffusion problems. J. Math. Sci. (N. Y.), 170(4):554-566, 2010. Problems in mathematical analysis. No. 50.

[47] H.-G. Roos, M. Stynes, and L. Tobiska. Numerical methods for singularly perturbed differential equations, volume 24 of Springer Series in Computational Mathematics. Springer-Verlag, Berlin, 1996. Convectiondiffusion and flow problems.

[48] O. Steinbach. Numerical approximation methods for elliptic boundary value problems. Springer, New York, 2008. Finite and boundary elements, Translated from the 2003 German original.

[49] O. Steinbach. Space-time finite element methods for parabolic problems. Computational Methods in Applied Mathematics, 15(4):551-566, 2015.

[50] Martin Stynes. Steady-state convection-diffusion problems. Acta Numer., 14:445-508, 2005.

[51] P. Sun, L. Chen, and J. Xu. In numerical studies of adaptive finite element methods for two dimensional convection-dominated problems. J. Sci. Comput., 43(1):24-43, 2010. 
[52] The FEniCS Project (C) Copyright 2015. The fenics project, 2015.

[53] R. R. P. van Nooyen. A Petrov-Galerkin mixed finite element method with exponential fitting. Numer. Methods Partial Differential Equations, 11(5):501-524, 1995.

[54] R. Verfürth. A review of a posteriori error estimation and adaptive mesh-refinement techniques. Wiley and Sons, Teubner, New-York, 1996.

[55] R. Verfürth. Robust a posteriori error estimates for stationary convection-diffusion equations. SIAM journal on numerical analysis, 43(4):1766-1782, 2005.

[56] J. Wloka. Partial Differential Equations. Cambridge University Press, 1987.

[57] J. Xu and L. Zikatanov. A monotone finite element scheme for convection-diffusion equations. Math. Comp., 68(228):1429-1446, 1999.

[58] Z.-R. Zhang and T. Tang. An adaptive mesh redistribution algorithm for convection-dominated problems. Commun. Pure Appl. Anal., 1(3):341-357, 2002.

[59] Z.M. Zhang. Finite element superconvergence on shishkin mesh for 2-d convection-diffusion problems. Math. Comput., 72(243):1147-1177, 2003. 